\title{
RiQueza florística del Complejo orográfico Sierra de NaJasa, provincia Camagüey, Cuba
}

\author{
Adelaida Barreto Valdes ${ }^{1}$, Daimy Godínez Caraballo ${ }^{1}$, \\ Néstor Enríquez Salgueiro ${ }^{1} \&$ Grisel Reyes Artiles ${ }^{1}$
}

\section{RESUMEN}

(Riqueza florística del complejo orográfico Sierra de Najasa, provincia Camagüey, Cuba) Las elevaciones que conforman el complejo orográfico Sierra de Najasa poseen diferentes categorías conservacionistas como parte del Sistema Provincial de Áreas Protegidas de la provincia de Camagüey. La necesidad de dar a conocer la riqueza de la flora que este encierra para establecer estrategias de conservación y manejo es objetivo de este trabajo. Se presenta la lista florística general que recoge 367 taxones de plantas vasculares, de 241 géneros y 75 familias. Fabaceae, Euphorbiaceae y Asteraceae son las más representadas. El $70.9 \%$ de los taxones se conocen por sus potencialidades como plantas medicinales, melíferas, maderables o de interés por otros usos. El endemismo se caracteriza por la presencia de 40 endémicos mayormente pancubanos. Se particulariza en las cinco elevaciones que caracterizan el mismo en lo referente a los aspectos más importantes como son: riqueza de taxones, riqueza por formaciones vegetales, endemismo y potencialidad económica de la flora.

Palabras clave: áreas protegidas, flora de Camagüey, Sierra de Najasa.

\section{Abstract}

(Floristic richness of Sierra de Najasa orographic complex, Camagüey's Province, Cuba) The elevations that are part of Sierra de Najasa orographic complex have different conservation categories as a part of Camagüey's Province Protected Area System. The objective of this work is the knowledge of their floristic richness in order to establish conservation strategies and management. A general list of the flora with 367 taxa of vascular plants, 241 genera and 75 families is presented; Fabaceae, Euphorbiaceae and Asteraceae are the most representative families. $70.9 \%$ of taxa are known by their potential as medicinal, melliferous and timber plants, or other uses. The endemism is characterized by the presence of 40 endemics, mostly pancubans. The more important aspects that characterize the five elevations are taxa richness, richness by types of vegetation, endemism and economic potential of the flora. Key words: protected areas, Camagüey's flora, Sierra of Najasa.

\section{INTRODUCCIÓN}

El municipio Najasa, situado al sur de la provincia Camagüey, tiene una extensión territorial de $921.44 \mathrm{~km}^{2}$, con las áreas mejor conservadas dentro del complejo orográfico Sierra de Najasa.

Estas elevaciones pertenecen, desde el punto de vista fitogeográfico, a la subprovincia florística Cuba Central, sector Cuba Centro Oriental, distrito Guaimarense; son montañas cársicas cubiertas por suelos húmico carbonáticos, en las que prevalece un clima tropical estacional (Borhidi \& Muñiz 1986).

La vegetación se caracteriza por los bosques semideciduos mesófilos y notófilos, el complejo de vegetación de mogote y las comunidades de sustitución o reemplazo (Ávila et al. 1987; Pérez et al. 1994; Primelles et al. 1999).
Es objetivo de este trabajo hacer énfasis en la riqueza florística de las alturas Sierra del Chorrillo, Sierra de Najasa, Sierra de Guaicanámar y Cerro Cachimbo que conforman el complejo orográfico (Fig. 1), para el trazado de estrategias de conservación y manejo de estas áreas como parte de la política ambiental del municipio.

\section{Materiales y Metodos}

Se revisaron los trabajos de Ávila et al. (1987), Barreto et al. (1992, 2002) y Pérez et al. (1994), que son fuente documental de los estudios florísticos realizados en la zona bajo análisis.

Se prepararon bases de datos computarizadas que incluyeron: la información existente, la obtenida a partir de los 15 días de recorridos y colectas hechos en el año 1997, y

Artigo recebido em 05/2005. Aceito para publicação em 06/2006.

${ }^{1}$ Centro de Investigaciones del Medio Ambiente, Camagüey, CITMA. Cisneros 105 altos e/ Angel y Pobre. 


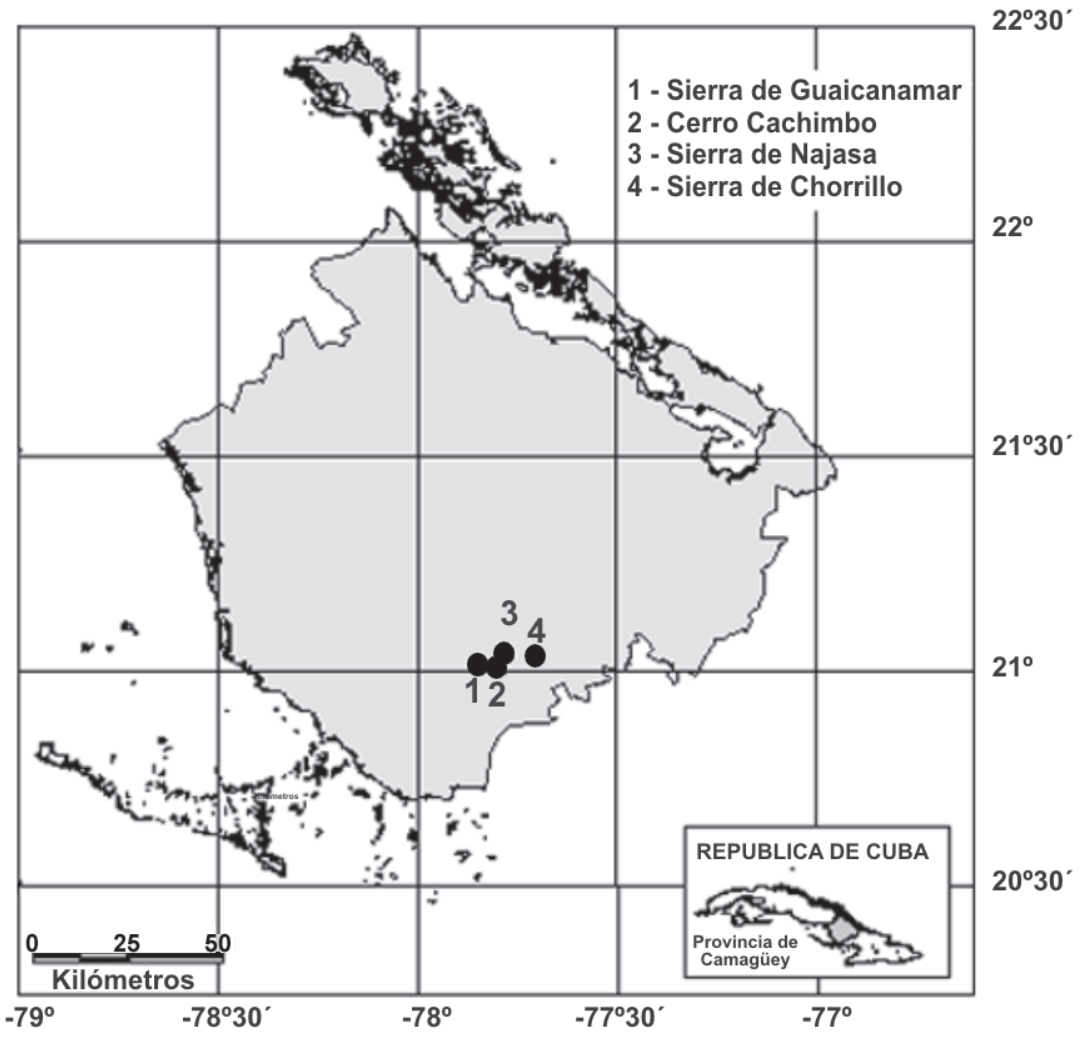

Figura 1 - Complejo Orográfico Sierra de Najasa.

aquella producto de la revisión de los ejemplares depositados en el herbario del Centro de Investigaciones de Medio Ambiente de Camagüey (HACC), lo que permitió un procesamiento ágil y eficaz y la obtención de las tablas y gráficos necesarios para su interpretación.

Las formaciones vegetales se trataron de acuerdo a Pérez et al. (1994) y los tipos de endémicos de Borhidi (1976).

Las potencialidades de la flora desde el punto de vista de los usos conocidos para cada taxon se tomaron de Fors (1957), Havard-Duclos (1969), Roig (1974), Ordext (1978), National Academy of Sciences (1979), Flores et al. (1988), Fuentes (1988), Hernández \& López (1991), Arias (1994) y Reyes et al. (2002).

\section{Resultados y Discusión}

La flora del complejo orográfico Sierra de Najasa se lista en la Tabla 1. Un total de 367 taxones, de 241 géneros, pertenecientes a 75 familias botánicas caracterizan la misma. Las familias más representadas son Fabaceae, Euphorbiaceae y Asteraceae con 38,18 y 14 , respectivamente.

La riqueza florística que aún prevalece en los ecosistemas boscosos que caracterizan las áreas trabajadas es interesante (Tab. 2 ), a pesar de las alteraciones en su estructura y composición de acuerdo a lo señalado por Ávila et al. (1987), Pérez et al. (1994) y Primelles et al. (1999). Las Sierras del Chorrillo y Najasa poseen el porciento más elevado de representatividad con relación a la flora general del complejo orográfico (Fig. 2), con 102 especies comunes de los bosques semideciduos (aproximadamente el 90\%) y el 38\% presente en éstos y en los bosques secundarios.

La Figura 3 muestra la riqueza por formaciones vegetales. Se aprecia que los bosques semideciduos mesófilos y notófilos muestran los valores más altos, esto es importante debido a que son las principales 


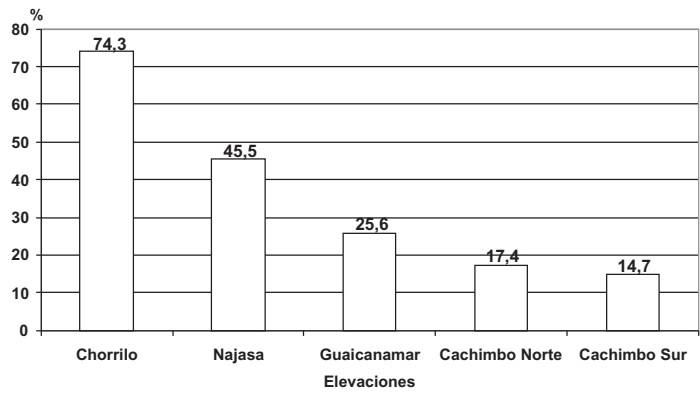

Figura 2 - Porciento de representatividad de las áreas respecto a la flora general del complejo.

unidades de vegetación que caracterizan cada una de las elevaciones estudiadas.

El endemismo lo conforman 40 taxones infragenéricos, lo que corresponde al $10.9 \%$ respecto a la flora del área, de los cuales 23 son pancubanos, dos de Cuba occidentalCuba central, cinco de Cuba central y diez de Cuba central-Cuba oriental.

En la Tabla 3 se observa que la Sierra del Chorrillo, aprobada como un Área Protegida de Recursos Manejados, posee el mayor índice de endemismo. Es de señalar, que esta sierra está conformada por las alturas mogotiformes Monte Quemado, La Belén y El Martillo, donde se encuentran exponentes como Dioscorea herradurensis y Rajania wilsoniana, de lugares muy específicos de las antiguas provincias político-administrativas de Pinar del Río y Las Villas, respectivamente, como expresaron Barreto et al. (1992). El aislamiento geográfico de estas estructuras cársicas y su difícil acceso ha permitido la conservación de los elementos autóctonos de la flora cubana que en ellas habitan, principalmente, en la formación vegetal de complejo de mogotes. Como especies propias del distrito fitogeográfico Guaimarense se encontraron Hidelgardia cubensis y Coccothrinax muricata, la primera en el extremo oriental de El Martillo y, la segunda en las otras alturas citadas.

La Sierra de Najasa categorizada como Paisaje Natural Protegido, es la segunda en importancia en cuanto a la presencia de taxones endémicos y de éstos, seis son comunes a la Sierra del Chorrillo, o sea el $40 \%$.

La Sierra de Guaicanámar, con la categoría conservacionista de Reserva Natural dentro del Sistema Provincial de Áreas Protegidas, y el Cerro Cachimbo, con la de Paisaje Natural Protegido, son las más pobres florísticamente y cuentan solamente con endémicos pancubanos, representados en las otras elevaciones (Tab. 1). Sus ecosistemas boscosos se han sometido a una intensa acción antrópica y para mantener estas categorías deben trazarse estrategias a nivel del gobierno municipal que inserten a las comunidades aledañas dentro de las mismas. Serían las encargadas de velar por el recurso natural que estas encierran y, con una acertada política de educación ambiental, podrían ayudar a la recuperación de sus ecosistemas y de sus valores paisajísticos.

Las potencialidades económicas de los taxones por área se recoge en la Tabla 4. El $70.9 \%$ tiene interés desde el punto de vista económico, por sus propiedades medicinales (214), melíferas (113), maderables (98) y con otras aplicaciones (138).

La necesidad de conservar la diversidad vegetal de este complejo orográfico se evidencia en los resultados obtenidos, y es

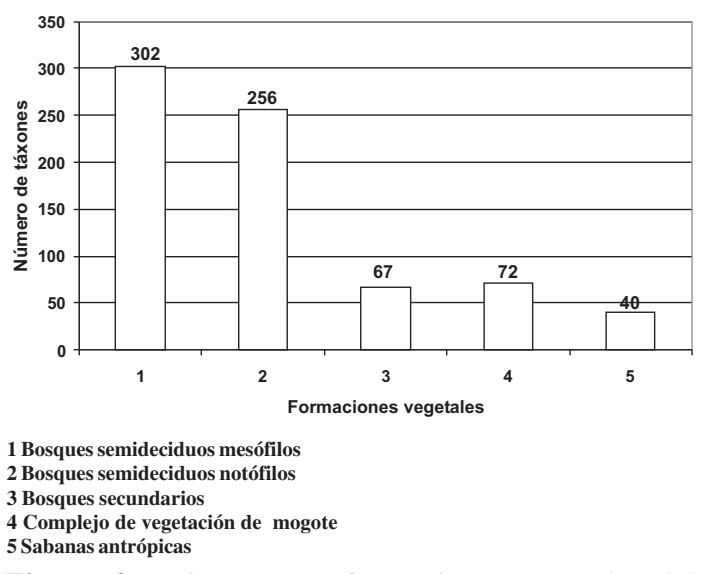

Figura 3 - Riqueza por formaciones vegetales del complejo 


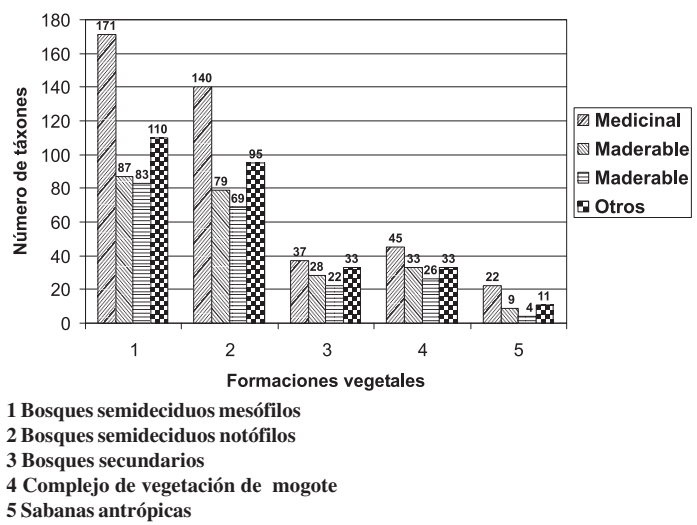

Figura 4 - Potencialidades por formaciones vegetales del complejo. interesante que en todas las elevaciones existe una flora de interés para propósitos diferentes, la cual está por encima del 70 porciento de representatividad de su riqueza florística. Es de destacar el Cerro Cachimbo Sur que posee el valor más alto. La Figura 4 muestra su comportamiento por formaciones vegetales; se aprecia que las más ricas en plantas útiles al hombre son los bosques semideciduos mesófilos y notófilos, lo que era de esperar por ser las de mayor riqueza florística.

Tabla 1 - Lista florística del complejo orográfico Sierra de Najasa. Tipo de Endémicos: PC: Pancubano, Coc-Cc: Cuba Occidental-Cuba central. Cc: Cuba Central, Cc-Cor: Cuba CentralCuba Oriental. C: Sierra del Chorrillo, N: Sierra de Najasa, G: Sierra de Guaicanámar, CN: Cerro Cachimbo (Norte), CS: Cerro Cachimbo (Sur). MD: medicinal; ML: melífera; MR: maderable; OA: otras aplicaciones. 1: bosque semideciduo mesófilo; 2: bosque semideciduo notófilo; 3: bosque secundario; 4: complejo de vegetación de mogote; 5: sabana antrópica.

\begin{tabular}{|c|c|c|c|c|c|c|c|c|c|c|c|c|c|c|c|c|}
\hline \multirow[t]{2}{*}{ Familia/Taxon } & \multirow[t]{2}{*}{ Nombre Común } & \multirow[t]{2}{*}{ End } & \multicolumn{5}{|c|}{ Elevaciones } & \multicolumn{4}{|c|}{ Usos } & \multicolumn{5}{|c|}{ Formaciones } \\
\hline & & & $\mathrm{C}$ & $\mathbf{N}$ & G & $\mathrm{CN}$ & CS & MD & ML & MR & $\mathrm{OA}$ & & eg & eta & les & \\
\hline \multicolumn{17}{|l|}{ ACANTHACEAE } \\
\hline Blechum pyramidatum (Lam.) Urb. & & & $\mathrm{X}$ & & & & & $\mathrm{X}$ & & & & 1 & 2 & & & \\
\hline Dicliptera vahliana Nees & Gallitos & & $\mathrm{X}$ & $\mathrm{X}$ & & & & & & & & 1 & 2 & & 4 & \\
\hline Oplonia tetrasticha (Wr. ex Griseb.) Stearn & No me toques & & $\mathrm{X}$ & & & & & & & & & 1 & 2 & & & \\
\hline Thunbergia alata Boj. ex Sims. & Ojo de poeta & & & $\mathrm{X}$ & & & & & & & & & 2 & 3 & & \\
\hline Thunbergia fragans Roxb. & Flor de nieve & & $\mathrm{X}$ & & & & & & & & & 1 & 2 & & & \\
\hline \multicolumn{17}{|l|}{ AGAVACEAE } \\
\hline Agave legrelliana Jacobi & & $\mathrm{Cc}$ & $\mathrm{X}$ & $\mathrm{X}$ & & & & $\mathrm{X}$ & & & $\mathrm{X}$ & 1 & 2 & & & \\
\hline \multicolumn{17}{|l|}{ AMARANTHACEAE } \\
\hline Achyranthes aspera var. indica Mill. & Bella María & & & & $\mathrm{X}$ & & & $\mathrm{X}$ & & & & 1 & & & & \\
\hline Alternanthera axillaris (Hornem.) D. Dietr. & Abrojo & $\mathrm{PC}$ & $\mathrm{X}$ & & & $\mathrm{X}$ & $\mathrm{X}$ & & & & & 1 & & & & 5 \\
\hline Alternanthera sessilis (L.) R. Br. ex DC. & Bella María & & & $\mathrm{X}$ & & & & & & & & & 2 & 3 & 4 & \\
\hline Chamissoa altissima (Jacq.) H.B.K. & Guaniquique & & $\mathrm{X}$ & & & & & $\mathrm{X}$ & & & $\mathrm{X}$ & 1 & 2 & & & \\
\hline Iresine angustifolia Euphrasen & & & $\mathrm{X}$ & & & & & & & & & 1 & & & & \\
\hline \multicolumn{17}{|l|}{ ANACARDIACEAE } \\
\hline Comocladia dentata Jacq. & Guao & & $\mathrm{X}$ & $\mathrm{X}$ & $\mathrm{X}$ & $\mathrm{X}$ & & $\mathrm{X}$ & & & $\mathrm{X}$ & 1 & 2 & 3 & & 5 \\
\hline Mangifera indica $\mathrm{L}$. & Mango & & $\mathrm{X}$ & & & & & $\mathrm{X}$ & $X$ & $\mathrm{X}$ & $\mathrm{X}$ & 1 & 2 & & & \\
\hline Spondias mombin L. & Jobo & & $\mathrm{X}$ & & & & & $\mathrm{X}$ & $X$ & $X$ & $\mathrm{X}$ & 1 & & & & \\
\hline \multicolumn{17}{|l|}{ ANNONACEAE } \\
\hline Annona reticulata $\mathrm{L}$. & Mamón & & & $\mathrm{X}$ & & & $\mathrm{X}$ & $X$ & & & $\mathrm{X}$ & & & & 4 & \\
\hline Oxandra lanceolata (Sw.) Baill. & Yaya & & $\mathrm{X}$ & $\mathrm{X}$ & $\mathrm{X}$ & $X$ & $\mathrm{X}$ & $\mathrm{X}$ & $X$ & $\mathrm{X}$ & $\mathrm{X}$ & 1 & 2 & 3 & 4 & \\
\hline \multicolumn{17}{|l|}{ APOCYNACEAE } \\
\hline Cameraria latifolia $\mathrm{L}$. & Maboa & & $\mathrm{X}$ & & & & & $\mathrm{X}$ & & $\mathrm{X}$ & & 1 & & & & \\
\hline Echites umbellata Jacq. & Curamagüey & & & $\mathrm{X}$ & & $\mathrm{X}$ & & $\mathrm{X}$ & & & $\mathrm{X}$ & & & & 4 & 5 \\
\hline Plumeria obtusa $\mathrm{L}$. & Lirio & & $\mathrm{X}$ & $\mathrm{X}$ & & & & $\mathrm{X}$ & & & & 1 & 2 & 3 & & \\
\hline Plumeria stenophylla Urb. & & Cc-Cor & $\mathrm{X}$ & & & & & $\mathrm{X}$ & & & & 1 & 2 & & & \\
\hline Rauvolfia salicifolia Griseb. & Corazón de paloma & Cc-Cor & $\mathrm{X}$ & & & & & $\mathrm{X}$ & & & & 1 & 2 & & & \\
\hline Rauvolfia tetraphylla $\mathrm{L}$. & Fruta de aura & & & $\mathrm{X}$ & & & & $\mathrm{X}$ & & & & & & & & 5 \\
\hline \multicolumn{17}{|l|}{ ARACEAE } \\
\hline Philodendron fragrantissimum (Hook.) G. Don & & & $\mathrm{X}$ & & & & & & & & & 1 & 2 & & & \\
\hline Philodendron consanguineum Schott & Macusey hembra & & $\mathrm{X}$ & & $\mathrm{X}$ & $\mathrm{X}$ & & & & & $\mathrm{X}$ & 1 & 2 & & & \\
\hline $\begin{array}{l}\text { Philodendron scandens ssp. cubense (Engl.) } \\
\text { I. Arias }\end{array}$ & Bejuco de lombrices & & $\mathrm{X}$ & & & & & & & & & 1 & 2 & & & \\
\hline
\end{tabular}




\begin{tabular}{|c|c|c|c|c|c|c|c|c|c|c|c|c|c|c|c|c|}
\hline \multirow{3}{*}{$\begin{array}{l}\text { Familia/Taxon } \\
\text { Philodendron lacerum (Jacq.) Schott }\end{array}$} & \multirow{3}{*}{$\begin{array}{l}\text { Nombre Común } \\
\text { Macusey macho }\end{array}$} & \multirow[t]{2}{*}{ End } & \multicolumn{5}{|c|}{ Elevaciones } & \multicolumn{4}{|c|}{ Usos } & \multirow{2}{*}{\multicolumn{5}{|c|}{$\begin{array}{c}\text { Formaciones } \\
\text { vegetales }\end{array}$}} \\
\hline & & & \multirow{2}{*}{$\frac{\mathrm{C}}{\mathrm{X}}$} & \multirow{2}{*}{$\begin{array}{l}\mathrm{N} \\
\mathrm{X}\end{array}$} & \multirow[t]{2}{*}{ G } & $\mathrm{CN}$ & \multirow[t]{2}{*}{ CS } & MD & & IL MR & $0 \mathrm{~A}$ & & & & & \\
\hline & & & & & & $\mathrm{X}$ & & & & & $\mathrm{X}$ & 1 & 2 & & & 5 \\
\hline Syngonium auritum (L.) Schott & & & $\mathrm{X}$ & $\mathrm{X}$ & & & & & & & $\mathrm{X}$ & 1 & 2 & & & \\
\hline Xanthosoma cubense (A. Rich.) Schott & Malanguilla & $\mathrm{PC}$ & $\mathrm{X}$ & & & & & & & & & 1 & & & & \\
\hline ARALIACEAE & & & & & & & & & & & & & & & & \\
\hline Dendropanax arboreus (L.) DC. \& Planch. & Víbona & & $\mathrm{X}$ & $\mathrm{X}$ & & & & $\mathrm{X}$ & & & & 1 & 2 & 3 & & \\
\hline $\begin{array}{l}\text { Schefflera morototoni (Aubl.) Maguire, } \\
\text { Steyermark et Frodin }\end{array}$ & Yagruma macho & & $\mathrm{X}$ & & & & & $\mathrm{X}$ & & $\mathrm{X}$ & & & 2 & & & \\
\hline ARECACEAE & & & & & & & & & & & & & & & & \\
\hline Coccothrinax muricata León & & $\mathrm{Cc}$ & $\mathrm{X}$ & & & & & & $\mathrm{X}$ & $\mathrm{X}$ & & 1 & 2 & & & \\
\hline Cocos nucifera $\mathrm{L}$ & Coco & & $\mathrm{X}$ & $\mathrm{X}$ & & & & $\mathrm{X}$ & $\mathrm{X}$ & & $\mathrm{X}$ & & 2 & & & \\
\hline Roystonea regia (H.B.K.) O.F.Cook var. regia & Palma real & & $\mathrm{X}$ & $\mathrm{X}$ & & & & $\mathrm{X}$ & $\mathrm{X}$ & $\mathrm{X}$ & $\mathrm{X}$ & 1 & 2 & 3 & 4 & 5 \\
\hline ASCLEPIADACEAE & & & & & & & & & & & & & & & & \\
\hline Asclepias curassavica $\mathrm{L}$. & Flor de calentura & & $\mathrm{X}$ & & & & & $\mathrm{X}$ & $\mathrm{X}$ & & & 1 & 2 & & & \\
\hline Asclepias nivea $\mathrm{L}$. & Flor de calentura & & $\mathrm{X}$ & & & & & $\mathrm{X}$ & $\mathrm{X}$ & & & 1 & 2 & & & \\
\hline Cynanchum brachystephanum (Griseb.) Alain & & $\mathrm{PC}$ & $\mathrm{X}$ & & & & & & & & & 1 & 2 & & & \\
\hline Cynanchum cubense (Griseb.) Woodson & & & $\mathrm{X}$ & & & & & & & & & 1 & 2 & & & \\
\hline Cynanchum sp. & & & & $\mathrm{X}$ & & & & & & & & 1 & & & & \\
\hline Fischeria crispiflora (Sw.) Schltr. & Curamagüey de costa & & $\mathrm{X}$ & & & & & & & & & 1 & 2 & & & \\
\hline Marsdenia clausa $\mathrm{R} . \mathrm{Br}$. & Curamagüey blanco & & $\mathrm{X}$ & $\mathrm{X}$ & & & & & & & & 1 & 2 & & & \\
\hline Oxypetalum cordifolium (Vent.) Schltr. & & & & $\mathrm{X}$ & & & & & & & & & 2 & & & \\
\hline Sarcostemma clausum (Jacq.) Roem. \& Schult. & & & & & $\mathrm{X}$ & & & & & & & 1 & & & & \\
\hline ASPLENIACEAE & & & & & & & & & & & & & & & & \\
\hline Asplenium dentatum $\mathrm{L}$. & Doradilla & & $\mathrm{X}$ & $\mathrm{X}$ & & & & & & & & 1 & 2 & 3 & & \\
\hline ASTERACEAE & & & & & & & & & & & & & & & & \\
\hline Bidens cynapiifolia H.B.K. & Romero amarillo & & $\mathrm{X}$ & & $\mathrm{X}$ & $\mathrm{X}$ & $\mathrm{X}$ & $\mathrm{X}$ & $\mathrm{X}$ & & $\mathrm{X}$ & 1 & 2 & & & \\
\hline Bidens pilosa $\mathrm{L}$. & Romerillo & & $\mathrm{X}$ & & & & $\mathrm{X}$ & $\mathrm{X}$ & $\mathrm{X}$ & & $\mathrm{X}$ & 1 & 2 & & & 5 \\
\hline Chromolaena odorata (L.) King \& Robins & Rompezaragüey falso & & & & $\mathrm{X}$ & & & $\mathrm{X}$ & $\mathrm{X}$ & & & 1 & & & & \\
\hline Elvira biflora (L.) DC. & & & $\mathrm{X}$ & & $\mathrm{X}$ & & & & & & & 1 & 2 & & & \\
\hline Eupatorium havanense H.B.K. & Rompezaragüey & & $\mathrm{X}$ & & & & & & & & & 1 & 2 & & & \\
\hline $\begin{array}{l}\text { Koanoplyllum villosum (Sw.) } \\
\text { R.M. King \& H. Rub. }\end{array}$ & Albahaca de sabana & & $\mathrm{X}$ & $\mathrm{X}$ & & $\mathrm{X}$ & & & & & & 1 & 2 & & & 5 \\
\hline Lagascea mollis Cav. & Romerillo cimarrón & & $\mathrm{X}$ & & & & & $\mathrm{X}$ & $\mathrm{X}$ & & & & 2 & & & \\
\hline Mikania micrantha H.B.K. var. micrantha & Guaco & & $\mathrm{X}$ & $\mathrm{X}$ & & & & $\mathrm{X}$ & & & & 1 & & & & 5 \\
\hline Parthenium hysterophorus L. & Escoba amarga & & $\mathrm{X}$ & & & & $\mathrm{X}$ & $\mathrm{X}$ & $\mathrm{X}$ & & & 1 & 2 & & & 5 \\
\hline Pluchea carolinensis Jacq. & Salvia de playa & & & $\mathrm{X}$ & & & & $\mathrm{X}$ & $X$ & & & & & & 4 & \\
\hline $\begin{array}{l}\text { Pseudoelephantopus spicatus } \\
\text { (B. Juss. ex Aubl.) C.F. Baker }\end{array}$ & Lengua de vaca & & $\mathrm{X}$ & $\mathrm{X}$ & & & & $\mathrm{X}$ & & & & 1 & 2 & 3 & & 5 \\
\hline Tridax procumbens $\mathrm{L}$. & Romerillo & & $\mathrm{X}$ & & & & & $\mathrm{X}$ & $\mathrm{X}$ & & & 1 & 2 & & & \\
\hline Trixis inula Crantz & Palo Santa María & & & $\mathrm{X}$ & & & & $\mathrm{X}$ & & & & & 2 & & 4 & \\
\hline Vernonia menthaefolia (Poepp. ex Spreng.) Less. & & $\mathrm{PC}$ & & $\mathrm{X}$ & & & & $\mathrm{X}$ & $\mathrm{X}$ & & & & 2 & & 4 & 5 \\
\hline BIGN & & & & & & & & & & & & & & & & \\
\hline Crescentia cujete $\mathrm{L}$. & Güira & & $\mathrm{X}$ & $\mathrm{X}$ & & & & $\mathrm{X}$ & $\mathrm{X}$ & $\mathrm{X}$ & $\mathrm{X}$ & 1 & 2 & 3 & & \\
\hline Cydista diversifolia (H.B.K.) Miers & Bejuco de vieja & & $\mathrm{X}$ & $\mathrm{X}$ & $\mathrm{X}$ & $\mathrm{X}$ & & $\mathrm{X}$ & $\mathrm{X}$ & & & 1 & 2 & & & \\
\hline Distictis gnaphalantha (A. Rich.) Urb. & & $\mathrm{PC}$ & $\mathrm{X}$ & $\mathrm{X}$ & $\mathrm{X}$ & $\mathrm{X}$ & $\mathrm{X}$ & $\mathrm{X}$ & & & & 1 & 2 & & 4 & \\
\hline Jacaranda coerulea (L.) Griseb. & Abey macho & & $\mathrm{X}$ & & & & & $\mathrm{X}$ & $X$ & $\mathrm{X}$ & & 1 & 2 & & & \\
\hline Pithecoctenium echinatum (Aubl.) K. Schum & Huevo de toro & & $\mathrm{X}$ & $\mathrm{X}$ & $\mathrm{X}$ & $\mathrm{X}$ & & & & & & 1 & 2 & 3 & 4 & \\
\hline Tabebuia angustata Britt. & Roble blanco & & $\mathrm{X}$ & & & & & $\mathrm{X}$ & $\mathrm{X}$ & $\mathrm{X}$ & $\mathrm{X}$ & 1 & & & & \\
\hline Tabebuia myrtifolia (Griseb.) Britt. & & $\mathrm{PC}$ & $\mathrm{X}$ & & & & & $\mathrm{X}$ & $\mathrm{X}$ & $\mathrm{X}$ & & 1 & 2 & & & \\
\hline BOMBACACEAE & & & & & & & & & & & & & & & & \\
\hline Ceiba pentandra (L.) Gaertn. & Ceiba & & $\mathrm{X}$ & $\mathrm{X}$ & $\mathrm{X}$ & & & $\mathrm{X}$ & $\mathrm{X}$ & $\mathrm{X}$ & $\mathrm{X}$ & 1 & 2 & 3 & 4 & \\
\hline BORAGINACEAE & & & & & & & & & & & & & & & & \\
\hline Bourreria cassinifolia (A. Rich.) Griseb. & Hierro de sabana & & $\mathrm{X}$ & & & & & $\mathrm{X}$ & & $\mathrm{X}$ & & 1 & & & & \\
\hline Bourreria cuneifolia O. E. Schulz & & $\mathrm{PC}$ & $\mathrm{X}$ & & & & & & & & & 1 & 2 & & & \\
\hline Bourreria virgata (Sw.) G. Don & Raspalengua & & $\mathrm{X}$ & & & & & & & & & 1 & 2 & & & \\
\hline Cordia collococca $\mathrm{L}$. & Ateje & & $\mathrm{X}$ & $\mathrm{X}$ & $\mathrm{X}$ & & $\mathrm{X}$ & & $\mathrm{X}$ & $\mathrm{X}$ & $\mathrm{X}$ & 1 & 2 & & 4 & \\
\hline Cordia gerascanthus $\mathrm{L}$. & Varía & & $\mathrm{X}$ & $\mathrm{X}$ & $\mathrm{X}$ & & $\mathrm{X}$ & $\mathrm{X}$ & $\mathrm{X}$ & $\mathrm{X}$ & $\mathrm{X}$ & 1 & 2 & 3 & 4 & \\
\hline $\begin{array}{l}\text { Cordia globosa (Jacq.) H.B.K. } \\
\text { var. humilis (Jacq.) Johnst. }\end{array}$ & Hierba de la sangre & & $\mathrm{X}$ & & & & & $\mathrm{X}$ & $\mathrm{X}$ & & & 1 & 2 & & & \\
\hline Ehretia tinifolia $\mathrm{L}$. & Roble prieto & & $\mathrm{X}$ & $\mathrm{X}$ & $\mathrm{X}$ & & & & & $\mathrm{X}$ & $\mathrm{X}$ & 1 & & & 4 & \\
\hline Heliotropium indicum $\mathrm{L}$. & Alacrancillo & & $\mathrm{X}$ & & & & & $\mathrm{X}$ & $\mathrm{X}$ & & $\mathrm{X}$ & 1 & 2 & & & \\
\hline Tournefortia hirsutissima $\mathrm{L}$. & Nigua & & & $\mathrm{X}$ & & & & $\mathrm{X}$ & $\mathrm{X}$ & & & & & 3 & & \\
\hline
\end{tabular}




\begin{tabular}{|c|c|c|c|c|c|c|c|c|c|c|c|c|c|c|c|}
\hline \multirow[t]{2}{*}{ Familia/Taxon } & \multirow[t]{2}{*}{ Nombre Común } & \multirow[t]{2}{*}{ End } & \multicolumn{5}{|c|}{ Elevaciones } & \multicolumn{4}{|c|}{ Usos } & \multirow{2}{*}{\multicolumn{4}{|c|}{$\begin{array}{c}\text { Formaciones } \\
\text { vegetales }\end{array}$}} \\
\hline & & & $\mathrm{C}$ & $\mathrm{N}$ & G & $\mathrm{CN}$ & CS & $\mathrm{MD}$ & & MR & $0 \mathrm{~A}$ & & & & \\
\hline \multicolumn{16}{|l|}{ BROMELIACEAE } \\
\hline Bromelia pinguin $\mathrm{L}$. & Piña de ratón & & $\mathrm{X}$ & & $\mathrm{X}$ & $\mathrm{X}$ & & $\mathrm{X}$ & & & $\mathrm{X}$ & 1 & & & \\
\hline Hohenbergia penduliflora (A. Rich.) Mez & & & $\mathrm{X}$ & & & & & & & & & 1 & 2 & & \\
\hline Tillandsia argentea Griseb. & & & & $\mathrm{X}$ & & $\mathrm{X}$ & & & & & & 1 & & & 5 \\
\hline Tillandsia balbisiana Schult. & & & & $\mathrm{X}$ & & & & $\mathrm{X}$ & & & & & 2 & & 5 \\
\hline Tillandsia fasciculata $\mathrm{Sw}$. & & & $X$ & & & $\mathrm{X}$ & & & & & & 1 & 2 & & 5 \\
\hline Tillandsia festucoides Brogn. ex Mez & & & & $\mathrm{X}$ & & & & & & & & & 2 & & \\
\hline Tillandsia flexuosa $\mathrm{Sw}$ & & & $\mathrm{X}$ & & & & & & & & $\mathrm{X}$ & 1 & 2 & & \\
\hline Tillandsia recurvata $\mathrm{L}$. & & & $\mathrm{X}$ & $\mathrm{X}$ & & & $\mathrm{X}$ & $\mathrm{X}$ & & & & 1 & 2 & & \\
\hline Tillandsia setacea $\mathrm{Sw}$. & & & & $\mathrm{X}$ & & & & & & & & & 2 & & \\
\hline Tillandsia tenuifolia $\mathrm{L}$. & & & $\mathrm{X}$ & & & & & & & & & 1 & 2 & & \\
\hline Tillandsia usneoides $\mathrm{L}$. & Guajaca & & $X$ & $\mathrm{X}$ & & & & $\mathrm{X}$ & & & $\mathrm{X}$ & 1 & 2 & & \\
\hline Tillandsia valenzuelana A. Rich. & & & $\mathrm{X}$ & $\mathrm{X}$ & & & & & & & $\mathrm{X}$ & 1 & 2 & & \\
\hline \multicolumn{16}{|l|}{ BURSERACEAE } \\
\hline Bursera simaruba (L.) Sargent. & Almácigo & & $\mathrm{X}$ & $\mathrm{X}$ & $\mathrm{X}$ & & & $\mathrm{X}$ & $\mathrm{X}$ & $X$ & $\mathrm{X}$ & 1 & 2 & & \\
\hline \multicolumn{16}{|l|}{ CACTACEAE } \\
\hline Harrisia eriophora (Pfeiff.) Britt. & Pitahaya & $\mathrm{PC}$ & & $\mathrm{X}$ & & & & & & & & 1 & & & \\
\hline Leptocereus maxonii Britt. \& Rose & & Cc-Cor & $\mathrm{X}$ & & & & & & & & & 1 & 2 & & \\
\hline Mamillaria prolifera (Mill.) Haw. & & & $\mathrm{X}$ & $\mathrm{X}$ & & & & & & & & 1 & 2 & & \\
\hline Pilosocereus brooksianus (Vaup.) Byl. \& Rowl. & Jíjara & Cc-Cor & $\mathrm{X}$ & & & & & $\mathrm{X}$ & & & & 1 & 2 & & \\
\hline Pilosocereus grandiflorus (L.) Britt. \& Rose & Jújira & Cc-Cor & & $\mathrm{X}$ & & & & $\mathrm{X}$ & & & & 1 & & & \\
\hline Rhipsalis baccifera (J. S. Mill.) Stearn. & Disciplinilla & & $\mathrm{X}$ & & & & & $\mathrm{X}$ & & & & 1 & & & \\
\hline Selenicereus boeckmannii (Otto.) Britt. \& Rose & & & & & & $\mathrm{X}$ & & & & & & 1 & & & \\
\hline Selenicereus grandiflorus (L.) Britt. \& Rose & Pitahaya & & $\mathrm{X}$ & $\mathrm{X}$ & & & & $\mathrm{X}$ & & & $\mathrm{X}$ & 1 & 2 & & \\
\hline \multicolumn{16}{|l|}{ CANELLACEAE } \\
\hline Canella alba Murray & Cúrbana & & $\mathrm{X}$ & $\mathrm{X}$ & $\mathrm{X}$ & & & $\mathrm{X}$ & $\mathrm{X}$ & $\mathrm{X}$ & $\mathrm{X}$ & 1 & 2 & 3 & \\
\hline \multicolumn{16}{|l|}{ CAPPARACEAE } \\
\hline Capparis cynophallophora L. & Mostacilla & & $\mathrm{X}$ & & & & & $\mathrm{X}$ & & $\mathrm{X}$ & $\mathrm{X}$ & 1 & 2 & & \\
\hline Capparis ferruginea $\mathrm{L}$. & Olivo & & $X$ & & & & & $\mathrm{X}$ & & $\mathrm{X}$ & & 1 & 2 & & \\
\hline Capparis flexuosa $\mathrm{L}$. & Palo barba de indio & & $\mathrm{X}$ & $\mathrm{X}$ & $\mathrm{X}$ & $\mathrm{X}$ & $\mathrm{X}$ & & & $\mathrm{X}$ & & 1 & 2 & & 5 \\
\hline Cleome spinosa Jacq. & Volantín & & $\mathrm{X}$ & & & & & $\mathrm{X}$ & $\mathrm{X}$ & & & 1 & 2 & & \\
\hline CARICACEAE & & & & & & & & & & & & & & & \\
\hline Carica papaya $\mathrm{L}$. & Fruta bomba & & $\mathrm{X}$ & & & $\mathrm{X}$ & $\mathrm{X}$ & $\mathrm{X}$ & $\mathrm{X}$ & & $\mathrm{X}$ & 1 & & & \\
\hline CECROPIACEAE & & & & & & & & & & & & & & & \\
\hline Cecropia peltata $L$. Miq. & Yagruma & & $\mathrm{X}$ & $\mathrm{X}$ & $\mathrm{X}$ & & & $\mathrm{X}$ & & $\mathrm{X}$ & $\mathrm{X}$ & 1 & 2 & 3 & 4 \\
\hline CELASTRACEAE & & & & & & & & & & & & & & & \\
\hline Crossopetalum aquifolium (Griseb.) A.S. Hitchc. & & & $\mathrm{X}$ & & & & & $\mathrm{X}$ & & & & 1 & 2 & & \\
\hline Crossopetalum uragoga (Jacq.) O. Kuntze & Hierba maravedí & & $\mathrm{X}$ & & & & & $\mathrm{X}$ & & & & 1 & 2 & & \\
\hline Cuervea integrifolia (A. Rich.) A. C. Sm. & Amansa guapo & $\mathrm{PC}$ & $\mathrm{X}$ & & & & & & & & & 1 & & & \\
\hline Maytenus buxifolia (A. Rich.) Griseb. & Carne de vaca & & $\mathrm{X}$ & & & & & $\mathrm{X}$ & & & & 1 & & & \\
\hline Schaefferia frutescens Jacq. & Cafecillo & & $\mathrm{X}$ & & & & & $\mathrm{X}$ & & & & 1 & & & \\
\hline CLUSIACEAE & & & & & & & & & & & & & & & \\
\hline Calophyllum antillanum Britt. & Ocuje & & $\mathrm{X}$ & $\mathrm{X}$ & & & & $\mathrm{X}$ & $\mathrm{X}$ & $\mathrm{X}$ & $\mathrm{X}$ & 1 & 2 & 3 & \\
\hline Garcinia bakeriana (Urb.) Borhidi & & $\mathrm{PC}$ & $\mathrm{X}$ & & & & & & & & & 1 & 2 & & \\
\hline COMMELINACEAE & & & & & & & & & & & & & & & \\
\hline Commelina diffusa Burm.f. & & & & & & $\mathrm{X}$ & & & & & & & & & 5 \\
\hline Commelina erecta $\mathrm{L}$. & & & $\mathrm{X}$ & & & & & $\mathrm{X}$ & & & $\mathrm{X}$ & 1 & 2 & & \\
\hline CONNARACEAE & & & & & & & & & & & & & & & \\
\hline Rourea glabra H.B.K. & & & & & $\mathrm{X}$ & & & & & & $\mathrm{X}$ & 1 & & & \\
\hline CONVOLVULACEAE & & & & & & & & & & & & & & & \\
\hline Ipomoea acuminata (Vahl) R. \& S. & Aguinaldo morado & & $\mathrm{X}$ & & & & & $\mathrm{X}$ & & & & 1 & 2 & & \\
\hline Ipomoea cf. nil (L.) Roth. & Aguinaldo azul claro & & & $\mathrm{X}$ & & & & & & & & & & 3 & \\
\hline Ipomoea hederifolia $\mathrm{L}$. & & & $\mathrm{X}$ & $\mathrm{X}$ & & & & & & & & 1 & 2 & 3 & 4 \\
\hline Ipomoea setifera Poir. & & & & & $\mathrm{X}$ & $\mathrm{X}$ & $\mathrm{X}$ & & & & & 1 & & & \\
\hline Ipomoea tiliacea (Willd.) Choisy & Marrullero & & $\mathrm{X}$ & & & & $\mathrm{X}$ & $\mathrm{X}$ & $\mathrm{X}$ & & & 1 & 2 & & \\
\hline Turbina corymbosa (L.) Raf. & Aguinaldo de pascua & & $\mathrm{X}$ & $\mathrm{X}$ & & & & $\mathrm{X}$ & $\mathrm{X}$ & & & 1 & 2 & & \\
\hline CUCURBITACEAE & & & & & & & & & & & & & & & \\
\hline Anguria ottoniana Schlecht. & & & $\mathrm{X}$ & & & & $\mathrm{X}$ & & & & & 1 & 2 & & 5 \\
\hline
\end{tabular}




\begin{tabular}{|c|c|c|c|c|c|c|c|c|c|c|c|c|c|c|c|c|}
\hline \multirow[t]{2}{*}{ Familia/Taxon } & \multirow[t]{2}{*}{ Nombre Común } & \multirow[t]{2}{*}{ End } & \multicolumn{5}{|c|}{ Elevaciones } & \multicolumn{4}{|c|}{ Usos } & \multirow{2}{*}{\multicolumn{5}{|c|}{$\begin{array}{c}\text { Formaciones } \\
\text { vegetales }\end{array}$}} \\
\hline & & & \multirow{2}{*}{$\begin{array}{l}\mathrm{C} \\
\mathrm{X}\end{array}$} & \multirow{2}{*}{$\begin{array}{l}\mathrm{N} \\
\mathrm{X}\end{array}$} & \multirow{3}{*}{$\begin{array}{l}\mathrm{G} \\
\mathrm{X}\end{array}$} & \multicolumn{2}{|c|}{$\mathrm{CN}$ CS } & \multirow{3}{*}{$\begin{array}{l}\text { MD } \\
X \\
X\end{array}$} & & MR & $\mathrm{OA}$ & & & & & \\
\hline Anguria pedata (L.) Jacq. & Pepino cimarrón & & & & & & & & & & & 1 & & 3 & & \\
\hline Melothria guadalupensis (Spreng.) Cogn. & & & & & & & & & & & $\mathrm{X}$ & & 2 & & & \\
\hline Momordica charantia L. & Cundeamor & & $X$ & $\mathrm{X}$ & $X$ & $\mathrm{X}$ & & $\mathrm{X}$ & $X$ & & & 1 & 2 & & 4 & \\
\hline CYPERACEAE & & & & & & & & & & & & & & & & \\
\hline Cyperus alternifolius $\mathrm{L}$. & Paragüita & & $X$ & & & & & $\mathrm{X}$ & & & & 1 & & & & \\
\hline DIOSCOREACEAE & & & & & & & & & & & & & & & & \\
\hline Dioscorea herradurensis (R. Knuth) P. Wilson & & Coc-Cc & $\mathrm{X}$ & & & & & & & & & 1 & 2 & & & \\
\hline Dioscorea polygonoides H. \& B. & & & $\mathrm{X}$ & & & & & & & & & 1 & 2 & & & \\
\hline Rajania angustifolia $\mathrm{Sw}$. & Name cimarrón & & $\mathrm{X}$ & & & & & & & & & 1 & 2 & & & \\
\hline Rajania cordata $\mathrm{L}$. & & & & & $X$ & & & & & & & 1 & & & & \\
\hline Rajania psilostachya (Knuth) Uline ex Knuth & & $\mathrm{PC}$ & $\mathrm{X}$ & & & & & $\mathrm{X}$ & & & & 1 & 2 & & & \\
\hline Rajania quinquefolia $\mathrm{L}$. & & & & & $X$ & & $X$ & & & & & 1 & & & & \\
\hline Rajania wilsoniana Morton & & $\mathrm{Cc}$ & $\mathrm{X}$ & & $X$ & & & & & & & 1 & & & & \\
\hline DRYOPTERIDACEAE & & & & & & & & & & & & & & & & \\
\hline Cyclopeltis semicordata (Sw.) J. Smith & & & & $\mathrm{X}$ & & & & & & & & & 2 & & & \\
\hline EBENACEAE & & & & & & & & & & & & & & & & \\
\hline Diospyros grisebachii (Hiern.) Standl. & Ebano real & $\mathrm{PC}$ & $X$ & $\mathrm{X}$ & & & & $\mathrm{X}$ & $X$ & $X$ & $X$ & 1 & 2 & 3 & & \\
\hline ERYTHROXYLACEAE & & & & & & & & & & & & & & & & \\
\hline Erythroxylum confusum Britt. & Arabo & & $\mathrm{X}$ & & & & & & $\mathrm{X}$ & $\mathrm{X}$ & & 1 & & & & \\
\hline Erythroxylum havanense Jacq. & Jibá & & $\mathrm{X}$ & $\mathrm{X}$ & $X$ & $\mathrm{X}$ & $\mathrm{X}$ & $\mathrm{X}$ & $\mathrm{X}$ & $\mathrm{X}$ & $\mathrm{X}$ & 1 & 2 & & 4 & 5 \\
\hline Erythroxylum rotundifolium Lunan & Arabillo & & $X$ & & & & & $X$ & $X$ & $\mathrm{X}$ & $X$ & 1 & 2 & & & \\
\hline EUPHORBIACEAE & & & & & & & & & & & & & & & & \\
\hline Acalypha alopecuroides Jacq. & Rabo de gato & & $\mathrm{X}$ & & & & & $\mathrm{X}$ & & & & 1 & 2 & & & \\
\hline Adelia ricinella $\mathrm{L}$. & Jía & & $\mathrm{X}$ & $\mathrm{X}$ & $\mathrm{X}$ & & & $\mathrm{X}$ & $\mathrm{X}$ & $\mathrm{X}$ & $\mathrm{X}$ & 1 & 2 & 3 & 4 & \\
\hline Ateramnus lucidus (Sw.) Rothm. & Yaití & & $\mathrm{X}$ & $\mathrm{X}$ & & & & $\mathrm{X}$ & $\mathrm{X}$ & $\mathrm{X}$ & $\mathrm{X}$ & 1 & 2 & & & \\
\hline Bernardia dichotoma (Willd.) Müll. Arg. & Cacapul & & $\mathrm{X}$ & & & & & & & & & 1 & 2 & & & \\
\hline Chamaesyce hirta (L.) Millsp. var. hirta & Lechera & & & & & & $\mathrm{X}$ & $\mathrm{X}$ & & & & & & & & 5 \\
\hline Croton lobatus L. & Frailecillo cimarrón & & $\mathrm{X}$ & & & & & $\mathrm{X}$ & & & & 1 & 2 & & & \\
\hline Croton lucidus L. & Cuabilla & & $\mathrm{X}$ & $\mathrm{X}$ & & & & $\mathrm{X}$ & & & & 1 & 2 & & 4 & \\
\hline Croton sagraeanus Müll. Arg. & Aceitillo & $\mathrm{PC}$ & & $\mathrm{X}$ & & & & $\mathrm{X}$ & $\mathrm{X}$ & & & 1 & & & & \\
\hline Cubanthus linearifolius (Griseb.) Millsp. & & Cc-Cor & $\mathrm{X}$ & & & & & & & & & 1 & 2 & & & \\
\hline Drypetes alba Poit. & Hueso & & $\mathrm{X}$ & & & & & & & $\mathrm{X}$ & & 1 & 2 & & & \\
\hline Drypetes lateriflora (Sw.) Krug \& Urb. & Hueso de monte & & $\mathrm{X}$ & & & & & & $\mathrm{X}$ & $\mathrm{X}$ & & 1 & & & & \\
\hline Drypetes mucronata Griseb. & Hueso de costa & & $\mathrm{X}$ & & & & & & & $\mathrm{X}$ & & 1 & 2 & & & \\
\hline Euphorbia heterophylla L. var. heterophylla & Corazón de María & & $\mathrm{X}$ & $\mathrm{X}$ & & & & & $\mathrm{X}$ & & & 1 & & & 4 & \\
\hline Grimmeodendron eglandulosum (A. Rich.) Urb. & Manzanillo & & $\mathrm{X}$ & & & & & $\mathrm{X}$ & & & & 1 & 2 & & & \\
\hline Margaritaria nobilis L. f. & Guaicaje & & $\mathrm{X}$ & & & & & & & & & 1 & & & & \\
\hline Platygyna hexandra (Jacq.) Müll. Arg. & Ortiguilla & $\mathrm{PC}$ & $\mathrm{X}$ & $\mathrm{X}$ & & & & $\mathrm{X}$ & & & $\mathrm{X}$ & 1 & 2 & 3 & 4 & \\
\hline Savia sessiliflora (Sw.) Willd. & Ahorca jíbaro & & $\mathrm{X}$ & & & & & & & $\mathrm{X}$ & & 1 & 2 & & & \\
\hline Tragia volubilis $\mathrm{L}$. & Candelilla & & $\mathrm{X}$ & $\mathrm{X}$ & $\mathrm{X}$ & & $\mathrm{X}$ & $\mathrm{X}$ & & & & 1 & 2 & & & \\
\hline FABACEAE-CAESALPINIOIDEAE & & & & & & & & & & & & & & & & \\
\hline Poeppigia procera Presl. & Tengue & & $\mathrm{X}$ & $\mathrm{X}$ & $\mathrm{X}$ & & & $\mathrm{X}$ & & $\mathrm{X}$ & $\mathrm{X}$ & 1 & 2 & 3 & 4 & \\
\hline Senna alata (L.) Roxb. & Guacamaya francesa & & $\mathrm{X}$ & & & & & $\mathrm{X}$ & $\mathrm{X}$ & & $\mathrm{X}$ & 1 & 2 & & & \\
\hline $\begin{array}{l}\text { Senna insularis (Britt. \& Rose) } \\
\text { Irwin et Barneby }\end{array}$ & Bejuco de la virgen & $\mathrm{PC}$ & $\mathrm{X}$ & & & & & $\mathrm{X}$ & & & & 1 & 2 & & & \\
\hline $\begin{array}{l}\text { Senna ligustrina }(\mathrm{L} .) \text { Irwin \& } \\
\text { Barneby var. ligustrina }\end{array}$ & Sen del país & & $\mathrm{X}$ & & & & & $\mathrm{X}$ & & & $\mathrm{X}$ & 1 & 2 & & & \\
\hline Senna obtusifolia (L.) Irwin \& Barneby & Guanina & & & & & $\mathrm{X}$ & & $\mathrm{X}$ & & & $\mathrm{X}$ & & & & & 5 \\
\hline Senna occidentalis (L.) Link & Platanillo & & & & & $\mathrm{X}$ & $\mathrm{X}$ & $\mathrm{X}$ & & & $\mathrm{X}$ & & & & & 5 \\
\hline $\begin{array}{l}\text { Senna spectabilis (DC.) Irwin \& Barneby } \\
\quad \text { var. spectabilis }\end{array}$ & Algarrobillo & & & $\mathrm{X}$ & & & $\mathrm{X}$ & $\mathrm{X}$ & & $\mathrm{X}$ & $\mathrm{X}$ & 1 & 2 & & 4 & 5 \\
\hline Senna uniflora (Mill.) Irwin \& Barneby & Guanina & & & & & $X$ & $\mathrm{X}$ & & & & & & & & & 5 \\
\hline FABACEAE-FABOIDEAE & & & & & & & & & & & & & & & & \\
\hline Aeschynomene americana $\mathrm{L}$. & Tamarindillo & & $\mathrm{X}$ & & $\mathrm{X}$ & $\mathrm{X}$ & & $\mathrm{X}$ & & & $\mathrm{X}$ & 1 & 2 & & & \\
\hline Andira inermis (W. Wright) Kunth ex DC. & Yaba & & $\mathrm{X}$ & & & & & $\mathrm{X}$ & $\mathrm{X}$ & $\mathrm{X}$ & $\mathrm{X}$ & 1 & 2 & & & \\
\hline Ateleia cubensis Griseb. var. cubensis & Rala de gallina & & $\mathrm{X}$ & $\mathrm{X}$ & & & & $\mathrm{X}$ & & & & 1 & 2 & 3 & 4 & \\
\hline Calopogonium coeruleum (Benth.) Hemsl. & Jícama dulce & & $\mathrm{X}$ & $\mathrm{X}$ & & & $\mathrm{X}$ & & $\mathrm{X}$ & & & 1 & 2 & 3 & 4 & 5 \\
\hline Canavalia rosea (Sw.) DC. & Mate colorado & $\mathrm{PC}$ & & $\mathrm{X}$ & $\mathrm{X}$ & & & $\mathrm{X}$ & & & $\mathrm{X}$ & 1 & 2 & 3 & & \\
\hline Centrosema molle Mart. ex Benth. & Bejuco de chivo & & $\mathrm{X}$ & $\mathrm{X}$ & & $\mathrm{X}$ & & & & & $\mathrm{X}$ & 1 & 2 & & 4 & 5 \\
\hline Centrosema plumieri (Turp. \& Pers.) Benth. & & & $\mathrm{X}$ & $\mathrm{X}$ & $X$ & $\mathrm{X}$ & $\mathrm{X}$ & $\mathrm{X}$ & & & $\mathrm{X}$ & 1 & 2 & & & \\
\hline Centrosema virginianum (L.) Benth. & Azulada & & $\mathrm{X}$ & $\mathrm{X}$ & $\mathrm{X}$ & & $\mathrm{X}$ & $\mathrm{X}$ & $\mathrm{X}$ & & $\mathrm{X}$ & 1 & 2 & & 4 & \\
\hline
\end{tabular}




\begin{tabular}{|c|c|c|c|c|c|c|c|c|c|c|c|c|c|c|c|c|}
\hline \multirow[t]{2}{*}{ Familia/Taxon } & \multirow[t]{2}{*}{ Nombre Común } & \multirow[t]{2}{*}{ End } & \multicolumn{5}{|c|}{ Elevaciones } & \multicolumn{4}{|c|}{ Usos } & \multirow{2}{*}{\multicolumn{5}{|c|}{$\begin{array}{c}\text { Formaciones } \\
\text { vegetales }\end{array}$}} \\
\hline & & & $\mathrm{C}$ & $\mathbf{N}$ & G & $\mathrm{CN}$ & CS & $\mathrm{MD}$ & MI & & $0 \mathrm{~A}$ & & & & & \\
\hline Crotalaria incana $\mathrm{L}$. & Garbancillo & & & $\mathrm{X}$ & & & & $\mathrm{X}$ & & & $\mathrm{X}$ & & & & 4 & \\
\hline Desmodium affine Schlecht. & Amor seco & & & & $\mathrm{X}$ & $\mathrm{X}$ & & & & & & & 2 & & & \\
\hline Desmodium axillare (Sw.) DC. var. axillare & Amor seco & & & & $\mathrm{X}$ & & & $\mathrm{X}$ & & & & 1 & 2 & & & \\
\hline Desmodium incanum DC. var. incanum & Amor seco & & $\mathrm{X}$ & $\mathrm{X}$ & $\mathrm{X}$ & $\mathrm{X}$ & & $\mathrm{X}$ & & & & 1 & 2 & & 4 & \\
\hline $\begin{array}{l}\text { Desmodium scorpiurus (Sw.) Desv. } \\
\text { var. scorpiurus }\end{array}$ & Amor seco & & & $\mathrm{X}$ & & $\mathrm{X}$ & & & & & & & 2 & & 4 & 5 \\
\hline Desmodium triflorum (L.) DC. & Amor seco & & & & & $\mathrm{X}$ & $X$ & $\mathrm{X}$ & & & $\mathrm{X}$ & & & & & 5 \\
\hline Galactia striata (Jacq.) Urb. & & & $\mathrm{X}$ & & $\mathrm{X}$ & & $\mathrm{X}$ & $\mathrm{X}$ & & $\mathrm{X}$ & $\mathrm{X}$ & 1 & 2 & & & \\
\hline Gliricidia sepium (Jacq.) Kunth ex Walp. & Piñón florido & & $\mathrm{X}$ & & & & & $\mathrm{X}$ & $\mathrm{X}$ & $\mathrm{X}$ & $\mathrm{X}$ & 1 & 2 & & & \\
\hline Hebestigma cubense (H.B.K.) Urb. & Frijolillo & & $\mathrm{X}$ & $\mathrm{X}$ & $\mathrm{X}$ & $\mathrm{X}$ & & & $\mathrm{X}$ & $\mathrm{X}$ & & 1 & 2 & 3 & 4 & 5 \\
\hline Mucuna pruriens (L.) DC. var. pruriens & Pica pica & & $\mathrm{X}$ & & $\mathrm{X}$ & & & $\mathrm{X}$ & & & $\mathrm{X}$ & 1 & 2 & & & \\
\hline \multicolumn{17}{|l|}{ FABACEAE-MIMOSOIDEAE } \\
\hline Abarema glauca (Urb.) Barneby \& J. W. Grimes & & & & $\mathrm{X}$ & & & & & $\mathrm{X}$ & & & & & 3 & & \\
\hline Acacia farnesiana (L.) Willd. & Aroma amarilla & & & $\mathrm{X}$ & $\mathrm{X}$ & $\mathrm{X}$ & $\mathrm{X}$ & $\mathrm{X}$ & $\mathrm{X}$ & & $\mathrm{X}$ & 1 & & & 4 & \\
\hline Acacia maschalocephala Griseb. & Tocino & $\mathrm{PC}$ & $\mathrm{X}$ & & $\mathrm{X}$ & & $\mathrm{X}$ & & & & & 1 & & & & \\
\hline Acacia tenuifolia (L.) Willd. & Tocino & & & $\mathrm{X}$ & & & & & & & & 1 & & & 4 & \\
\hline Desmanthus virgatus (L.) Willd. & Adormidera & & $\mathrm{X}$ & $\mathrm{X}$ & $\mathrm{X}$ & & & $\mathrm{X}$ & & & & 1 & & & & \\
\hline Dichrostachys cinerea (L.) Wight \& Arn. & Marabú & & & & $\mathrm{X}$ & & & $\mathrm{X}$ & $\mathrm{X}$ & $\mathrm{X}$ & $\mathrm{X}$ & 1 & & & & \\
\hline Lysiloma sabicu Benth. & Sabicú & & $\mathrm{X}$ & $\mathrm{X}$ & & & & & & $\mathrm{X}$ & & 1 & 2 & & 4 & \\
\hline Mimosa pudica $\mathrm{L}$. & Dormidera & & $\mathrm{X}$ & & $\mathrm{X}$ & $\mathrm{X}$ & $\mathrm{X}$ & $\mathrm{X}$ & & & $\mathrm{X}$ & 1 & 2 & & & 5 \\
\hline $\begin{array}{l}\text { Pseudoalbizia berteriana } \\
\quad \text { (Balbis ex DC.) Britt. \& Rose }\end{array}$ & Abey blanco & & $\mathrm{X}$ & & & & & & & $\mathrm{X}$ & & 1 & 2 & & & \\
\hline Samanea saman (Jacq.) Merr. & Algarrobo & & $\mathrm{X}$ & $\mathrm{X}$ & $\mathrm{X}$ & & $\mathrm{X}$ & & $\mathrm{X}$ & $\mathrm{X}$ & $\mathrm{X}$ & 1 & 2 & 3 & 4 & \\
\hline Zapoteca formosa (Kunth) H.M. Hern. & & & $\mathrm{X}$ & $\mathrm{X}$ & $\mathrm{X}$ & $\mathrm{X}$ & & & $\mathrm{X}$ & $\mathrm{X}$ & $\mathrm{X}$ & 1 & 2 & 3 & 4 & \\
\hline \multicolumn{17}{|l|}{ FLACOURTIACEAE } \\
\hline Casearia aculeata Jacq. & Jía brava & & $\mathrm{X}$ & $\mathrm{X}$ & $\mathrm{X}$ & $\mathrm{X}$ & $\mathrm{X}$ & $\mathrm{X}$ & $\mathrm{X}$ & $\mathrm{X}$ & & 1 & 2 & & 4 & \\
\hline Casearia guianensis (Aubl.) Urb. & Jía amarilla & & $\mathrm{X}$ & $\mathrm{X}$ & & & & & & $\mathrm{X}$ & & 1 & 2 & & 4 & \\
\hline Casearia sylvestris $\mathrm{Sw}$. var. sylvestris & Sarnilla & & $\mathrm{X}$ & & & & & $\mathrm{X}$ & $\mathrm{X}$ & $\mathrm{X}$ & & 1 & 2 & & & \\
\hline Gossypiospermum eriophorum (C.Wr.) Urb. & Agracejo & & $\mathrm{X}$ & & $\mathrm{X}$ & & & $\mathrm{X}$ & $\mathrm{X}$ & & & 1 & 2 & & & \\
\hline Zuelania guidonia (Sw.) Britt. \& Millsp. & Guaguasí & & $\mathrm{X}$ & & & & & $\mathrm{X}$ & & $\mathrm{X}$ & $\mathrm{X}$ & 1 & 2 & & & \\
\hline \multicolumn{17}{|l|}{ LAMIACEAE } \\
\hline Hyptis verticillata Jacq. & Jardinera & & $\mathrm{X}$ & & & & & $\mathrm{X}$ & $\mathrm{X}$ & & & 1 & 2 & & & \\
\hline Hyptis pectinata (L.) Poit. & Alhucema & & & $\mathrm{X}$ & & & & $\mathrm{X}$ & $\mathrm{X}$ & & & & 2 & & & \\
\hline Hyptis suaveolens (L.) Poit. & & & & & $\mathrm{X}$ & & $\mathrm{X}$ & $\mathrm{X}$ & $\mathrm{X}$ & & & 1 & & & & \\
\hline \multicolumn{17}{|l|}{ LAURACEAE } \\
\hline Licaria triandria (Sw.) Kosterm. & Leviza & & $\mathrm{X}$ & $\mathrm{X}$ & & & & & $\mathrm{X}$ & $\mathrm{X}$ & $\mathrm{X}$ & 1 & & 3 & & \\
\hline Nectandra coriacea (Sw.) Griseb. & Cigua & & $\mathrm{X}$ & $\mathrm{X}$ & $\mathrm{X}$ & $\mathrm{X}$ & & $\mathrm{X}$ & & $\mathrm{X}$ & $\mathrm{X}$ & 1 & 2 & 3 & 4 & \\
\hline Persea americana Mill. var. americana & Aguacate & & $\mathrm{X}$ & & & & & $\mathrm{X}$ & & & $\mathrm{X}$ & 1 & 2 & & & \\
\hline LOGANIACEAE & & & & & & & & & & & & & & & & \\
\hline Strychnos grayi Griseb. & Manca montero & & $\mathrm{X}$ & & & & & & & & & 1 & 2 & & & \\
\hline MALPIGHIACEAE & & & & & & & & & & & & & & & & \\
\hline Bunchosia media (Ait.) DC. & Mierda gallina & & $\mathrm{X}$ & & & & & & & & & 1 & 2 & & & \\
\hline Bunchosia swartziana Griseb. & & & $\mathrm{X}$ & $\mathrm{X}$ & & & & & & & & 1 & & 3 & & \\
\hline Malpighia aquifolia $\mathrm{L}$. & & & $\mathrm{X}$ & & & & & & & & & 1 & 2 & & & \\
\hline Malpighia cnide Spreng. & Palo bronco & & $\mathrm{X}$ & & & & & & & & & & 2 & & & \\
\hline Malpighia martinicense Jacq. & & & $\mathrm{X}$ & & & & & & & & & 1 & 2 & & & \\
\hline Malpighia suberosa Small & Palo bronco & Cc-Cor & & $\mathrm{X}$ & & & & & & & & & & & 4 & \\
\hline Stigmaphyllon diversifolium (Kunth) A. Juss. & Bejuco blanco & & & $\mathrm{X}$ & & & & & & & & & 2 & 3 & & \\
\hline Stigmaphyllon sagraeanum A. Juss. & Bejuco San Pedro & & $\mathrm{X}$ & & $\mathrm{X}$ & & & $\mathrm{X}$ & & & & 1 & 2 & & & \\
\hline Triopteris rigida $\mathrm{Sw}$. & Amansa guapo & & $\mathrm{X}$ & & $\mathrm{X}$ & & $\mathrm{X}$ & $\mathrm{X}$ & & & & 1 & 2 & & & \\
\hline MALVACEAE & & & & & & & & & & & & & & & & \\
\hline Hibiscus tiliaceus L. & Majagua & & $\mathrm{X}$ & & & & & & $\mathrm{X}$ & $\mathrm{X}$ & $\mathrm{X}$ & 1 & & & & \\
\hline Malvastrum coromandelianum (L.) Garcke & Malva negra & & $\mathrm{X}$ & & & & & $\mathrm{X}$ & & & & 1 & & & & \\
\hline Pavonia fruticosa (Mill.) Fawc. \& Rendle & Tábano & & $\mathrm{X}$ & $\mathrm{X}$ & & & & $\mathrm{X}$ & & & & 1 & 2 & 3 & & \\
\hline Pavonia spinifex (L.) Cav. & Majagüilla de costa & & $\mathrm{X}$ & $\mathrm{X}$ & $\mathrm{X}$ & & & $\mathrm{X}$ & & & & 1 & 2 & & 4 & \\
\hline Sida acuminata DC. & & & $\mathrm{X}$ & & & & & & $\mathrm{X}$ & & & & 2 & & & \\
\hline Sida acuta Burm. & Malva de caballo & & & & $\mathrm{X}$ & $\mathrm{X}$ & & $\mathrm{X}$ & $\mathrm{X}$ & & $\mathrm{X}$ & 1 & & & & \\
\hline Sida glutinosa Cav. & Malva de Cuba & & & $\mathrm{X}$ & & & & $\mathrm{X}$ & $\mathrm{X}$ & & & & & & 4 & \\
\hline Sida pyramidata Cav. & & & & $\mathrm{X}$ & & & & & $\mathrm{X}$ & & & & & 3 & & \\
\hline Sida rhombifolia L. & Malva de cochino & & & $\mathrm{X}$ & & & & $\mathrm{X}$ & $\mathrm{X}$ & & & & & & 4 & \\
\hline Sida spinosa $\mathrm{L}$. & & & & $\mathrm{X}$ & & & $\mathrm{X}$ & & $\mathrm{X}$ & & $\mathrm{X}$ & & 2 & & & 5 \\
\hline
\end{tabular}




\begin{tabular}{|c|c|c|c|c|c|c|c|c|c|c|c|c|c|c|c|}
\hline \multirow[t]{2}{*}{ Familia/Taxon } & \multirow[t]{2}{*}{ Nombre Común } & \multirow[t]{2}{*}{ End } & \multicolumn{5}{|c|}{ Elevaciones } & \multicolumn{4}{|c|}{ Usos } & \multirow{2}{*}{\multicolumn{4}{|c|}{$\begin{array}{c}\text { Formaciones } \\
\text { vegetales }\end{array}$}} \\
\hline & & & $\mathrm{C}$ & $\mathbf{N}$ & G & & CS & MD & ML & & $\mathrm{OA}$ & & & & \\
\hline \multicolumn{16}{|l|}{ MELIACEAE } \\
\hline Cedrela odorata L. & Cedro & & $\mathrm{X}$ & $\mathrm{X}$ & $\mathrm{X}$ & & & $\mathrm{X}$ & $\mathrm{X}$ & $\mathrm{X}$ & $\mathrm{X}$ & 1 & 2 & & 4 \\
\hline Guarea guidonia (L.) Sleumer & Yamagua & & $X$ & $\mathrm{X}$ & & & & $\mathrm{X}$ & $\mathrm{X}$ & $\mathrm{X}$ & $\mathrm{X}$ & 1 & 2 & & 4 \\
\hline Swietenia mahagoni (L.) Jacq. & Caoba de Cuba & & $\mathrm{X}$ & $\mathrm{X}$ & & & & $\mathrm{X}$ & $\mathrm{X}$ & $\mathrm{X}$ & $\mathrm{X}$ & 1 & 2 & & 4 \\
\hline Trichilia havanensis Jacq. & Siguaraya & & $\mathrm{X}$ & $\mathrm{X}$ & & & & $\mathrm{X}$ & $\mathrm{X}$ & $\mathrm{X}$ & $\mathrm{X}$ & 1 & 2 & 3 & 4 \\
\hline Trichilia hirta $\mathrm{L}$. & Cabo de hacha & & $X$ & $\mathrm{X}$ & $X$ & $\mathrm{X}$ & $X$ & & $X$ & $\mathrm{X}$ & $\mathrm{X}$ & 1 & 2 & & 4 \\
\hline \multicolumn{16}{|l|}{ MENISPERMACEAE } \\
\hline Cissampelos pareira L. & Bejuco terciopelo & & $X$ & & & & & $\mathrm{X}$ & & & $\mathrm{X}$ & 1 & 2 & & \\
\hline Hyperbaena dominguensis (DC.) Benth. & & & $\mathrm{X}$ & & $\mathrm{X}$ & & & & & & & 1 & & & \\
\hline Hyperbaena racemosa Urb. & Chicharrón & $\mathrm{PC}$ & $\mathrm{X}$ & $\mathrm{X}$ & & & & & & & & 1 & 2 & & 4 \\
\hline \multicolumn{16}{|l|}{ MORACEAE } \\
\hline Chlorophora tinctoria (L.) Gaud. & Mora del país & & $\mathrm{X}$ & & & & & & & $\mathrm{X}$ & $\mathrm{X}$ & 1 & & & \\
\hline Ficus aurea Nutt. & Jagüey hembra & & $\mathrm{X}$ & $\mathrm{X}$ & & & & & & $\mathrm{X}$ & & 1 & 2 & 3 & \\
\hline Ficus maxima P. Mill. & Jagüey de río & & $X$ & & & & & & & $\mathrm{X}$ & & 1 & 2 & & \\
\hline Ficus membranacea $\mathrm{C}$. Wr. & Jagüey & & $\mathrm{X}$ & & & & & & & $\mathrm{X}$ & & 1 & 2 & & \\
\hline Ficus trigonata $\mathrm{L}$. & Jagüey & & $\mathrm{X}$ & & & & & $\mathrm{X}$ & & $\mathrm{X}$ & & 1 & & & \\
\hline \multicolumn{16}{|l|}{ MYRTACEAE } \\
\hline Eugenia axillaris (Sw.) Willd. & Guairaje & & $\mathrm{X}$ & & & & & $\mathrm{X}$ & $\mathrm{X}$ & $\mathrm{X}$ & $\mathrm{X}$ & 1 & 2 & & \\
\hline Eugenia ligustrina (Sw.) Willd. & Arraiján & & $\mathrm{X}$ & & $\mathrm{X}$ & & & $\mathrm{X}$ & & $\mathrm{X}$ & $\mathrm{X}$ & 1 & 2 & & \\
\hline Eugenia maleolens Poir. & & & & $\mathrm{X}$ & & & & $\mathrm{X}$ & & $\mathrm{X}$ & & & & 3 & \\
\hline Myrciaria floribunda (West. ex Willd.) Berg. & Mije & & & $\mathrm{X}$ & & & & & & $\mathrm{X}$ & $\mathrm{X}$ & & & 3 & \\
\hline Psidium guajava $\mathrm{L}$. & Guayaba & & $\mathrm{X}$ & & & & & $\mathrm{X}$ & $\mathrm{X}$ & $\mathrm{X}$ & $\mathrm{X}$ & 1 & & & \\
\hline \multicolumn{16}{|l|}{ NYCTAGINACEAE } \\
\hline Guapira discolor (Spreng.) Little & Barrchorno & & $\mathrm{X}$ & & & & & $\mathrm{X}$ & & $\mathrm{X}$ & & 1 & 2 & & \\
\hline Neea shaferi Standl. & & Cc-Cor & $\mathrm{X}$ & & & & & $\mathrm{X}$ & & & & 1 & & & \\
\hline Pisonia aculeata $\mathrm{L}$. & Zarza & & $\mathrm{X}$ & $\mathrm{X}$ & $\mathrm{X}$ & $\mathrm{X}$ & $\mathrm{X}$ & $\mathrm{X}$ & $X$ & $\mathrm{X}$ & $X$ & 1 & 2 & & \\
\hline OCHNACEAE & & & & & & & & & & & & & & & \\
\hline Ouratea ilicifolia (DC.) Baillon var. ilicifolia & Rascabarriga & & $\mathrm{X}$ & & & & & $X$ & & & & 1 & 2 & & \\
\hline ORCHIDACEAE & & & & & & & & & & & & & & & \\
\hline Encyclia fucata (Lindl.) Britton \& Millsp. & Flor de San Pedro & & $\mathrm{X}$ & & & & & $\mathrm{X}$ & & & & & 2 & & \\
\hline Epidendrum anceps Jacq. & & & $\mathrm{X}$ & & & & & & & & & 1 & & & \\
\hline Maxillaria crassifolia (Lindl.) Rchb.f. & & & $\mathrm{X}$ & & & & & & & & & 1 & 2 & & \\
\hline Oeceoclades maculata (Lindl.) Lindl. & & & & $\mathrm{X}$ & $\mathrm{X}$ & $X$ & & & & & & 1 & 2 & 3 & 4 \\
\hline Oncidium undulatum (Sw.) Salisbury & Oreja de burro & & $\mathrm{X}$ & & & & & & & & & 1 & 2 & & \\
\hline Stenorrhynchus lanceolatus (Aubl.) L.C. Rich. & & & $\mathrm{X}$ & & & & & & & & & 1 & 2 & & \\
\hline Vanilla dilloniana Correll & & & $\mathrm{X}$ & & & & & $\mathrm{X}$ & & & $\mathrm{X}$ & 1 & 2 & & \\
\hline Vanilla phaeantha Rchb. f. & & & $\mathrm{X}$ & $\mathrm{X}$ & & & & $\mathrm{X}$ & & & & 1 & 2 & & \\
\hline Vanilla $\mathrm{sp}$ & & & & & & $\mathrm{X}$ & & & & & & 1 & & & \\
\hline PASSIFLORACEAE & & & & & & & & & & & & & & & \\
\hline Passiflora capsularis $\mathrm{L}$. & & & & $\mathrm{X}$ & & & & & $X$ & & & & 2 & & 4 \\
\hline Passiflora cubensis $\mathrm{L}$. & Gürito de pasión & $\mathrm{PC}$ & & $\mathrm{X}$ & & & & $\mathrm{X}$ & $X$ & & $\mathrm{X}$ & 1 & & & \\
\hline Passiflora foetida $\mathrm{L}$. & Pasionaria & & & & & & $\mathrm{X}$ & $\mathrm{X}$ & & & & 1 & & & \\
\hline Passiflora multiflora $\mathrm{L}$. & Fruta de perro & & $X$ & & & & & & $X$ & & & 1 & & & \\
\hline Passiflora suberosa $\mathrm{L}$. & Huevo de gallo & & $\mathrm{X}$ & $\mathrm{X}$ & $\mathrm{X}$ & $\mathrm{X}$ & $\mathrm{X}$ & $\mathrm{X}$ & $\mathrm{X}$ & & & 1 & 2 & & 4 \\
\hline PHYTOLACACEAE & & & & & & & & & & & & & & & \\
\hline Petiveria alliacea $\mathrm{L}$. & Anamú & & $X$ & & $\mathrm{X}$ & $X$ & $\mathrm{X}$ & $\mathrm{X}$ & $X$ & & & 1 & 2 & & \\
\hline Rivina humilis L. & Coralitos & & $\mathrm{X}$ & $\mathrm{X}$ & $\mathrm{X}$ & & & $\mathrm{X}$ & $X$ & & $\mathrm{X}$ & 1 & 2 & 3 & \\
\hline Trichostigma octandrum (L.) H. Walt. & Bejuco canasta & & $\mathrm{X}$ & $\mathrm{X}$ & $\mathrm{X}$ & & & $\mathrm{X}$ & & & $\mathrm{X}$ & 1 & 2 & & \\
\hline PICRAMNIACEAE & & & & & & & & & & & & & & & \\
\hline Picramnia pentandra Sw. & Aguedita & $\mathrm{X}$ & $\mathrm{X}$ & $\mathrm{X}$ & $\mathrm{X}$ & & & $\mathrm{X}$ & $\mathrm{X}$ & $\mathrm{X}$ & $\mathrm{X}$ & 1 & 2 & 3 & \\
\hline PIPERACEAE & & & & & & & & & & & & & & & \\
\hline Peperomia petiolaris C. DC. & & $\mathrm{PC}$ & $\mathrm{X}$ & & & & & & & & & 1 & 2 & & \\
\hline Peperomia urocarpa Fisch \& Mey. & & & $\mathrm{X}$ & & & & & & & & & 1 & 2 & & \\
\hline Piper aduncum ssp. ossanum (C. DC.) Trel. & Platanillo de Cuba & $\mathrm{Coc}-\mathrm{Cc}$ & & $\mathrm{X}$ & & & & $\mathrm{X}$ & & & & 1 & 2 & & 4 \\
\hline Piper amalago $\mathrm{L}$. & Mataguao & & $\mathrm{X}$ & $\mathrm{X}$ & & & & $\mathrm{X}$ & & & $\mathrm{X}$ & 1 & 2 & 3 & \\
\hline Piper peltatum $\mathrm{L}$. & Caisimón & & & $\mathrm{X}$ & & & & & & & & & & 3 & \\
\hline Potomorphe umbellata (L.) Miq. & Caisimón & & $\mathrm{X}$ & & & & & & & & & & 2 & & \\
\hline PLUMBAGINACEAE & & & & & & & & & & & & & & & \\
\hline Plumbago scandens $\mathrm{L}$. & Malacara & & $\mathrm{X}$ & $\mathrm{X}$ & $\mathrm{X}$ & $\mathrm{X}$ & & $\mathrm{X}$ & & & & 1 & 2 & & 5 \\
\hline
\end{tabular}




\begin{tabular}{|c|c|c|c|c|c|c|c|c|c|c|c|c|c|c|}
\hline \multirow[t]{2}{*}{ Familia/Taxon } & \multirow[t]{2}{*}{ Nombre Común } & \multirow[t]{2}{*}{ End } & \multicolumn{5}{|c|}{ Elevaciones } & \multicolumn{4}{|c|}{ Usos } & \multirow{2}{*}{\multicolumn{3}{|c|}{$\begin{array}{c}\text { Formaciones } \\
\text { vegetales }\end{array}$}} \\
\hline & & & $\mathrm{C}$ & $\mathrm{N}$ & G & $\mathrm{CN}$ & CS & MD & ML & MR & $0 \mathrm{~A}$ & & & \\
\hline \multicolumn{15}{|l|}{ POACEAE } \\
\hline Eleusine indica (L.) Gaertn. & Pata de gallina & & $\mathrm{X}$ & & & & & $\mathrm{X}$ & & & $\mathrm{X}$ & 1 & 2 & \\
\hline Lasiacis divaricata (L.) Hitchc. & Pitillo de monte & & $\mathrm{X}$ & $\mathrm{X}$ & $\mathrm{X}$ & & & $\mathrm{X}$ & & & $\mathrm{X}$ & 1 & 2 & 4 \\
\hline Olyra latifolia $\mathrm{L}$. & Tibisí & & & $\mathrm{X}$ & $\mathrm{X}$ & $X$ & & $\mathrm{X}$ & & & $\mathrm{X}$ & 1 & 2 & 3 \\
\hline Oplismenus hirtellus (L.) Beauv. & & & & & $\mathrm{X}$ & & $\mathrm{X}$ & & & & $\mathrm{X}$ & 1 & 2 & \\
\hline Oplismenus setarius (Lam.) Roem. \& Schult. & Pitillo & & $\mathrm{X}$ & & & & & & & & $X$ & 1 & 2 & \\
\hline Panicum maximum Jacq. & Hierba guinea & & $\mathrm{X}$ & & & & & $\mathrm{X}$ & & & $\mathrm{X}$ & 1 & 2 & \\
\hline Paspalum notatum Flügge & Hierba tejana & & $\mathrm{X}$ & & & & & & & & $\mathrm{X}$ & 1 & 2 & \\
\hline Paspalum virgatum $\mathrm{L}$. & Caguazo & & $X$ & & & & & & & & $\mathrm{X}$ & 1 & & \\
\hline Pharus lappulaceus Aubl. & Guizazo de perro & & $\mathrm{X}$ & & & & & & & & & 1 & 2 & \\
\hline Pharus latifolius L. & & & $\mathrm{X}$ & & & & & & & & & 1 & 2 & \\
\hline Rhynchelytrum repens (Willd.) Hubbard & Hierba del Natal & & $\mathrm{X}$ & & & & & $\mathrm{X}$ & & & $\mathrm{X}$ & 1 & & \\
\hline \multicolumn{15}{|l|}{ POLYPODIACEAE } \\
\hline Campyloneurum phyllitides (L.) Presl. & Pasa de negro & & $\mathrm{X}$ & & & & & $X$ & & & & 1 & & \\
\hline Microgramma heterophylla (L.) Wherry & & & & $\mathrm{X}$ & & & & & & & & & & 3 \\
\hline \multicolumn{15}{|l|}{ POLYGALACEAE } \\
\hline Securidaca elliptica Turcz. & Maravedí & $\mathrm{PC}$ & $\mathrm{X}$ & & & & & $\mathrm{X}$ & & & & 1 & 2 & \\
\hline Securidaca lamarckii Griseb. & Flor de la cruz & & & $\mathrm{X}$ & & & & & & & & & & 5 \\
\hline Securidaca sp. & & & & & & $\mathrm{X}$ & & & & & & 1 & & \\
\hline \multicolumn{15}{|l|}{ PTERIDACEAE } \\
\hline Adiantum capillus-veneris $\mathrm{L}$. & Culantrillo de pozo & & & & $\mathrm{X}$ & $\mathrm{X}$ & $\mathrm{X}$ & $X$ & & & & 1 & & \\
\hline Adiantum melanoleucum Willd. & & & & $\mathrm{X}$ & & & & & & & & & 2 & 3 \\
\hline Adiantum pyramidale (L.) Willd. & & & & $\mathrm{X}$ & & & & & & & & & 2 & 4 \\
\hline Adiantum sp. & & & & & & $\mathrm{X}$ & & & & & & 1 & & \\
\hline Adiantum villosum $\mathrm{L}$. & & & $\mathrm{X}$ & & & & & & & & & 1 & & \\
\hline Asplenium jenmanii Proctor & & & & & & $\mathrm{X}$ & & & & & & 1 & & \\
\hline Cheylanthes microphylla (Sw.) Sw. & & & & $\mathrm{X}$ & & & & & & & & 1 & & \\
\hline \multicolumn{15}{|l|}{ RHAMNACEAE } \\
\hline Colubrina arborescens (Mill.) Sarg. & Bijáguara & & $\mathrm{X}$ & & & & & $\mathrm{X}$ & $\mathrm{X}$ & $\mathrm{X}$ & $\mathrm{X}$ & 1 & 2 & \\
\hline Colubrina elliptica (Sw.) Brizicki \& Stern & Jayabico & & $\mathrm{X}$ & $\mathrm{X}$ & & & & $\mathrm{X}$ & & $\mathrm{X}$ & $\mathrm{X}$ & 1 & 2 & 3 \\
\hline Gouania lupuloides (L.) Urb. var. lupuloides & Jaboncillo & & $\mathrm{X}$ & $\mathrm{X}$ & $\mathrm{X}$ & $\mathrm{X}$ & $\mathrm{X}$ & $\mathrm{X}$ & & & $\mathrm{X}$ & 1 & 2 & 34 \\
\hline Gouania polygama (Jacq.) Urb. & Jaboncillo & & $\mathrm{X}$ & $\mathrm{X}$ & & $\mathrm{X}$ & & $\mathrm{X}$ & $\mathrm{X}$ & & $\mathrm{X}$ & 1 & 2 & 4 \\
\hline RUBIACEAE & & & & & & & & & & & & & & \\
\hline Calycophyllum candidissimum (Vahl) DC. & Dágame & & $\mathrm{X}$ & & & & & $\mathrm{X}$ & $\mathrm{X}$ & $\mathrm{X}$ & $\mathrm{X}$ & 1 & 2 & \\
\hline Chiococca alba (L.) Hitchc. & Bejuco de verraco & & $\mathrm{X}$ & $\mathrm{X}$ & & & & $\mathrm{X}$ & & & & 1 & & 3 \\
\hline Erithalis fruticosa $\mathrm{L}$. & Cuaba prieta & & $\mathrm{X}$ & & & & & $\mathrm{X}$ & & & & 1 & & \\
\hline Faramea occidentalis (L.) A. Rich. & Nabaco & & $\mathrm{X}$ & & & & & $X$ & & & & 1 & 2 & \\
\hline Genipa americana $\mathrm{L}$. & Jagua & & $\mathrm{X}$ & & & & & $\mathrm{X}$ & $\mathrm{X}$ & $\mathrm{X}$ & $\mathrm{X}$ & 1 & 2 & \\
\hline Guettarda calyptrata A. Rich. & Guayabillo & $\mathrm{PC}$ & $\mathrm{X}$ & & & & & $\mathrm{X}$ & & $\mathrm{X}$ & & 1 & 2 & \\
\hline Guettarda elliptica $\mathrm{Sw}$. & Cigüilla & & $\mathrm{X}$ & & & & & & & & & 1 & 2 & \\
\hline Guettarda macrocarpa Griseb. & & Cc-Cor & $\mathrm{X}$ & & & & & & & & & 1 & 2 & \\
\hline Hamelia patens Jacq. & Ponasí & & $\mathrm{X}$ & $\mathrm{X}$ & & & & $\mathrm{X}$ & $\mathrm{X}$ & & $\mathrm{X}$ & 1 & & \\
\hline Psychotria horizontalis Sw. & & & $\mathrm{X}$ & & & & & & $\mathrm{X}$ & & $\mathrm{X}$ & 1 & 2 & \\
\hline Spermacoce assurgens Ruiz \& Pav. & Hierba de garro & & & $\mathrm{X}$ & & & & & & & & & & 4 \\
\hline RUTACEAE & & & & & & & & & & & & & & \\
\hline Amyris balsamifera $\mathrm{L}$. & Cuaba & & $\mathrm{X}$ & & & & & $\mathrm{X}$ & $\mathrm{X}$ & $\mathrm{X}$ & $\mathrm{X}$ & 1 & 2 & \\
\hline Amyris elemifera $\mathrm{L}$. & Cuaba amarilla de costa & & $\mathrm{X}$ & $\mathrm{X}$ & & & & $\mathrm{X}$ & & $\mathrm{X}$ & & 1 & 2 & \\
\hline Citrus aurantium $\mathrm{L}$. & Naranja agria & & $\mathrm{X}$ & & & & $\mathrm{X}$ & $\mathrm{X}$ & $X$ & $\mathrm{X}$ & $\mathrm{X}$ & 1 & & 5 \\
\hline Citrus limon (L.) Burm. f. & Limón & & & $\mathrm{X}$ & $\mathrm{X}$ & & & $\mathrm{X}$ & $\mathrm{X}$ & $\mathrm{X}$ & $\mathrm{X}$ & 1 & 2 & 3 \\
\hline Citrus sinensis (L.) Osbeck & Naranja dulce & & $\mathrm{X}$ & $\mathrm{X}$ & & & & $\mathrm{X}$ & & & $\mathrm{X}$ & 1 & 2 & \\
\hline Zanthoxylum martinicense (Lam.) DC. & Ayúa & & $\mathrm{X}$ & $\mathrm{X}$ & $\mathrm{X}$ & $\mathrm{X}$ & & & $\mathrm{X}$ & $\mathrm{X}$ & $\mathrm{X}$ & 1 & 2 & 34 \\
\hline SAPINDACEAE & & & & & & & & & & & & & & \\
\hline Allophyllus racemosa $\mathrm{Sw}$. & Palo de caja & & $\mathrm{X}$ & & & & & & & & & 1 & 2 & \\
\hline $\begin{array}{l}\text { Cardiospermum halicacabum } \\
\text { L. var. halicacabum }\end{array}$ & Farolito & & $\mathrm{X}$ & & & & & $\mathrm{X}$ & & & & 1 & & \\
\hline Cupania americana $\mathrm{L}$. & Guara común & & $\mathrm{X}$ & $\mathrm{X}$ & & $\mathrm{X}$ & & $\mathrm{X}$ & $\mathrm{X}$ & $\mathrm{X}$ & $\mathrm{X}$ & 1 & 2 & 4 \\
\hline Cupania glabra Sw. var. glabra & Guara de costa & & $\mathrm{X}$ & $\mathrm{X}$ & $\mathrm{X}$ & & & $\mathrm{X}$ & $\mathrm{X}$ & $\mathrm{X}$ & $\mathrm{X}$ & 1 & 2 & 3 \\
\hline Cupania macrophylla A. Rich. & Guara blanca & & $\mathrm{X}$ & & & & & $\mathrm{X}$ & $\mathrm{X}$ & $\mathrm{X}$ & $\mathrm{X}$ & 1 & 2 & \\
\hline Hypelate trifoliata $\mathrm{Sw}$. & Hueso de costa & & $\mathrm{X}$ & & & & & $\mathrm{X}$ & & $\mathrm{X}$ & $\mathrm{X}$ & 1 & & \\
\hline Melicoccus bijugatus Jacq. & Mamoncillo & & & $\mathrm{X}$ & $\mathrm{X}$ & $\mathrm{X}$ & $\mathrm{X}$ & $\mathrm{X}$ & $\mathrm{X}$ & $\mathrm{X}$ & $\mathrm{X}$ & 1 & 2 & \\
\hline Paullinia fuscescens H.B.K. & Bejuco de vieja & & $\mathrm{X}$ & $\mathrm{X}$ & $\mathrm{X}$ & & & & & & $\mathrm{X}$ & 1 & 2 & 34 \\
\hline
\end{tabular}




\begin{tabular}{|c|c|c|c|c|c|c|c|c|c|c|c|c|c|c|c|c|}
\hline \multirow[t]{2}{*}{ Familia/Taxon } & \multirow[t]{2}{*}{ Nombre Común } & \multirow[t]{2}{*}{ End } & \multicolumn{5}{|c|}{ Elevaciones } & \multicolumn{4}{|c|}{ Usos } & \multirow{2}{*}{\multicolumn{5}{|c|}{$\begin{array}{c}\text { Formaciones } \\
\text { vegetales }\end{array}$}} \\
\hline & & & \multirow[t]{2}{*}{$\mathrm{C}$} & \multirow{2}{*}{$\frac{\mathrm{N}}{\mathrm{X}}$} & \multirow[t]{2}{*}{ G } & \multicolumn{2}{|c|}{$\mathrm{CN}$ CS } & \multirow[t]{2}{*}{ MD } & \multicolumn{3}{|c|}{ ML MR OA } & & & & & \\
\hline Paullinia jamaicensis Macf. & Bejuco matancero & & & & & & & & & & & & & 3 & & \\
\hline Serjania diversifolia (Jacq.) Radlk. & Bejuco colorado & & & & $\mathrm{X}$ & & & $\mathrm{X}$ & & & & 1 & 2 & & & \\
\hline Thouinia trifoliata Poit. & Negra Cuba & & $\mathrm{X}$ & & & & & $\mathrm{X}$ & & $\mathrm{X}$ & & 1 & 2 & & & \\
\hline SAPOTACEAE & & & & & & & & & & & & & & & & \\
\hline Chrysophyllum oliviforme $\mathrm{L}$. & Caimitillo & & $\mathrm{X}$ & $\mathrm{X}$ & $X$ & & & $\mathrm{X}$ & $\mathrm{X}$ & $\mathrm{X}$ & $\mathrm{X}$ & 1 & 2 & & 4 & \\
\hline $\begin{array}{l}\text { Pouteria dominigensis (C.F.Gaertn.) Baehni } \\
\text { Baehni ssp. dominigensis }\end{array}$ & Sapote culebra & & $\mathrm{X}$ & & & & & & & $\mathrm{X}$ & $\mathrm{X}$ & 1 & 2 & & & \\
\hline $\begin{array}{l}\text { Sideroxylon foetidissimum Jacq. } \\
\text { ssp. foetidissimum }\end{array}$ & Jocúma & & $\mathrm{X}$ & $\mathrm{X}$ & $\mathrm{X}$ & & & $X$ & & $\mathrm{X}$ & $X$ & 1 & 2 & 3 & 4 & \\
\hline Sideroxylon salicifolium (L.) C.F. Gaertner & Cuyá & & $X$ & & $\mathrm{X}$ & $\mathrm{X}$ & & $\mathrm{X}$ & $\mathrm{X}$ & $\mathrm{X}$ & $\mathrm{X}$ & 1 & 2 & & 5 & \\
\hline SCHIZAEACEAE & & & & & & & & & & & & & & & & \\
\hline Anemia adiantifolia (L.) Sw. & & & & $X$ & & & & & & & & & 2 & & 4 & \\
\hline SIMAROUBACEAE & & & & & & & & & & & & & & & & \\
\hline Simarouba glauca var. latifolia Cronquist & Simaruba & & $\mathrm{X}$ & & & & & $\mathrm{X}$ & $\mathrm{X}$ & $\mathrm{X}$ & $\mathrm{X}$ & 1 & 2 & & & \\
\hline Simarouba glauca var. typica Cronquist & Gavilán & & & $\mathrm{X}$ & $\mathrm{X}$ & & & $\mathrm{X}$ & & & & 1 & 2 & & & \\
\hline SMILACACEAE & & & & & & & & & & & & & & & & \\
\hline Smilax domingensis Willd. & Raíz de china & & & $\mathrm{X}$ & & & & & $X$ & & $\mathrm{X}$ & & & 3 & & \\
\hline Smilax havanensis Jacq. & Bejuco ñame & & $\mathrm{X}$ & $\mathrm{X}$ & & & & $X$ & $\mathrm{X}$ & & $X$ & 1 & 2 & 3 & & \\
\hline SOLANACEAE & & & & & & & & & & & & & & & & \\
\hline Capsicum frutescens $\mathrm{L}$. & Ají guaguao & & $\mathrm{X}$ & $\mathrm{X}$ & $\mathrm{X}$ & & $\mathrm{X}$ & $\mathrm{X}$ & & & $\mathrm{X}$ & 1 & 2 & & & \\
\hline Solandra grandiflora $\mathrm{Sw}$. & Chamico bejuco & & $X$ & & & & & $\mathrm{X}$ & & & & 1 & 2 & & & \\
\hline Solanum erianthum D. Don & Pendejera macho & & & $\mathrm{X}$ & $\mathrm{X}$ & $X$ & & $\mathrm{X}$ & & & & 1 & & 3 & & \\
\hline Solanum havanense Jacq. & Lila & & $\mathrm{X}$ & $\mathrm{X}$ & $X$ & & & & & & $\mathrm{X}$ & 1 & 2 & 3 & & \\
\hline Solanum torvum $\mathrm{Sw}$. & Pendejera & & & $\mathrm{X}$ & & & & $\mathrm{X}$ & & & & 1 & & 3 & & \\
\hline STERCULIACEAE & & & & & & & & & & & & & & & & \\
\hline Guazuma ulmifolia Lam. & Guásima & & $\mathrm{X}$ & $\mathrm{X}$ & $\mathrm{X}$ & $\mathrm{X}$ & $\mathrm{X}$ & $\mathrm{X}$ & $\mathrm{X}$ & $\mathrm{X}$ & $\mathrm{X}$ & 1 & 2 & & 4 & \\
\hline Hildergardia cubensis (Urb.) Kosterm. & Guana & Cc-Cor & $\mathrm{X}$ & $\mathrm{X}$ & & & & & & $\mathrm{X}$ & $\mathrm{X}$ & 1 & 2 & & & \\
\hline Melochia nodiflora $\mathrm{Sw}$. & Malva colorada & & & $\mathrm{X}$ & & & & $\mathrm{X}$ & $\mathrm{X}$ & & & & & 3 & & \\
\hline Melochia pyramidata L. var. pyramidata & Malva común & & & $\mathrm{X}$ & & & & $\mathrm{X}$ & & & & & 2 & & & \\
\hline Sterculia apetala (Jacq.) Karst. & Anacagüita & & & $\mathrm{X}$ & $\mathrm{X}$ & & $\mathrm{X}$ & $\mathrm{X}$ & $\mathrm{X}$ & $\mathrm{X}$ & $\mathrm{X}$ & 1 & & & & \\
\hline Waltheria indica $\mathrm{L}$. & Malva & & & & & & $\mathrm{X}$ & $\mathrm{X}$ & $\mathrm{X}$ & & & & & & & 5 \\
\hline THELYPTERIDACEAE & & & & & & & & & & & & & & & & \\
\hline $\begin{array}{l}\text { Thelypteris patens var. scabriuscula } \\
\text { (Presl.) A.R. Smith }\end{array}$ & & & & $\mathrm{X}$ & & & & & & & & & & & 4 & \\
\hline Thelypteris tetragona (Sw.) Small var. tetragona & & & & $\mathrm{X}$ & & & & & & & & & & 3 & & \\
\hline THEOPHRASTACEAE & & & & & & & & & & & & & & & & \\
\hline Jacquinia aculeata (L.) Mez & Espuela de caballero & $\mathrm{PC}$ & $\mathrm{X}$ & & $\mathrm{X}$ & & & $\mathrm{X}$ & & $\mathrm{X}$ & $\mathrm{X}$ & 1 & 2 & & & \\
\hline TILIACEAE & & & & & & & & & & & & & & & & \\
\hline Corchorus siliquosus L. & Malva té & & & $\mathrm{X}$ & $\mathrm{X}$ & $\mathrm{X}$ & $\mathrm{X}$ & $\mathrm{X}$ & $\mathrm{X}$ & & $\mathrm{X}$ & 1 & & & 4 & \\
\hline Luehea speciosa Willd. & & & $\mathrm{X}$ & & & & & & & $\mathrm{X}$ & & & 2 & & & \\
\hline Triumfetta semitriloba Jacq. & Guizazo & & $\mathrm{X}$ & $\mathrm{X}$ & & & $\mathrm{X}$ & $\mathrm{X}$ & $\mathrm{X}$ & & & 1 & 2 & 3 & 4 & 5 \\
\hline ULMACEAE & & & & & & & & & & & & & & & & \\
\hline Ampelocera cubensis Griseb. & Jatía blanca & & $\mathrm{X}$ & & & & & & & $\mathrm{X}$ & & 1 & 2 & & & \\
\hline Ampelocera pubescens Morton & & $\mathrm{Cc}$ & $\mathrm{X}$ & & & & & & & & & 1 & & & & \\
\hline Celtis iguanaea (Jacq.) Sarg. & Zarza blanca & & $\mathrm{X}$ & & & & & $\mathrm{X}$ & & & $\mathrm{X}$ & 1 & 2 & & & \\
\hline Celtis trinervia Lam. & Ramón de sierra & & $\mathrm{X}$ & $\mathrm{X}$ & $\mathrm{X}$ & $\mathrm{X}$ & $\mathrm{X}$ & & $\mathrm{X}$ & $\mathrm{X}$ & $\mathrm{X}$ & 1 & 2 & & & \\
\hline Phyllostylon brasiliensis Capanema & Jatía & & $\mathrm{X}$ & & & & & $\mathrm{X}$ & & $\mathrm{X}$ & & 1 & & & & \\
\hline URTICACEAE & & & & & & & & & & & & & & & & \\
\hline Pilea depressa (Sw.) Blume & & & $\mathrm{X}$ & & & & & & & & & 1 & 2 & & & \\
\hline Pilea herniarioides (Sw.) Lindl. & & & $\mathrm{X}$ & & & & & & & & & 1 & 2 & & & \\
\hline Pilea trianthemoides (Sw.) Lindl. & & & & $\mathrm{X}$ & & & & & & & $\mathrm{X}$ & & & 3 & & \\
\hline Urera baccifera (L.) Gaud. & Cichicate & & & & & $\mathrm{X}$ & & $\mathrm{X}$ & & & & & & & & 5 \\
\hline VERBENACEAE & & & & & & & & & & & & & & & & \\
\hline Bouchea prismatica $(\mathrm{L}$.$) Kuntze var. prismatica$ & Verbena cimarrona & & $\mathrm{X}$ & & & & $\mathrm{X}$ & $\mathrm{X}$ & & & & 1 & & & & 5 \\
\hline Citharexylum fruticosum L. var. fruticosum & Guayo blanco & & $\mathrm{X}$ & & & & & & $\mathrm{X}$ & $\mathrm{X}$ & $\mathrm{X}$ & 1 & 2 & & & \\
\hline Lantana camara L. var. camara & Filigrana & & & $\mathrm{X}$ & & & & $\mathrm{X}$ & $\mathrm{X}$ & & $\mathrm{X}$ & & & 3 & & \\
\hline Priva lappulacea (L.) Pers. & Farolito & & $\mathrm{X}$ & $\mathrm{X}$ & $\mathrm{X}$ & $\mathrm{X}$ & & $\mathrm{X}$ & & & & 1 & 2 & & & \\
\hline Stachytarpheta jamaicensis (L.) Vahl & Verbena azul & & $\mathrm{X}$ & $\mathrm{X}$ & $\mathrm{X}$ & & & $\mathrm{X}$ & & & & 1 & 2 & & 4 & \\
\hline
\end{tabular}




\begin{tabular}{|c|c|c|c|c|c|c|c|c|c|c|c|c|}
\hline \multirow[t]{2}{*}{ Familia/Taxon } & \multirow[t]{2}{*}{ Nombre Común } & \multirow[t]{2}{*}{ End } & \multicolumn{5}{|c|}{ Elevaciones } & \multicolumn{2}{|r|}{ Usos } & \multirow{2}{*}{\multicolumn{3}{|c|}{$\begin{array}{c}\text { Formaciones } \\
\text { vegetales }\end{array}$}} \\
\hline & & & $\mathrm{C}$ & $\mathbf{N}$ & G & $\mathrm{CN}$ & CS & MD & ML MR OA & & & \\
\hline Verbena scabra Vahl & Verbena cimarrona & & & $\mathrm{X}$ & & & & & & & & 45 \\
\hline \multicolumn{13}{|l|}{ VIOLACEAE } \\
\hline Hybanthus havanensis Jacq. & Hierba de San Martin & & $\mathrm{X}$ & & & & $\mathrm{X}$ & $\mathrm{X}$ & & 1 & 2 & 5 \\
\hline \multicolumn{13}{|l|}{ VITACEAE } \\
\hline Cissus torreana Britt. \& Wils. & & $\mathrm{Cc}$ & $\mathrm{X}$ & & & & & & & 1 & 2 & \\
\hline Cissus verticillata (L.) Nicolson \& Jar. & Bejuco ubí & & $\mathrm{X}$ & & $\mathrm{X}$ & $\mathrm{X}$ & & $\mathrm{X}$ & $\mathrm{X}$ & 1 & 2 & \\
\hline
\end{tabular}

Tabla 2 - Riqueza florística de las elevaciones del complejo orográfico.

\begin{tabular}{lllrrc}
\hline Elevaciones Altitud(m.s.n.m.) & Familias & Géneros & Taxones & $\begin{array}{c}\text { \% representatividad respecto } \\
\text { a las plantas vasculares cubanas }\end{array}$ \\
\hline Sierra del Chorrillo & 301 & 71 & 207 & 273 & 4.1 \\
Sierra de Najasa & 312 & 64 & 132 & 167 & 2.5 \\
Sierra Guaicanámar & 253.1 & 49 & 82 & 94 & 1.4 \\
Cerro Cachimbo (N) & 191.3 & 40 & 55 & 64 & 0.9 \\
Cerro Cachimbo (S) & 188.4 & 33 & 47 & 54 & 0.8 \\
\hline
\end{tabular}

Tabla 3 - Endemismo por elevación estudiada. PC: pancubano, Coc-CC: Cuba occidental-Cuba central, CC: Cuba Central, Cc-Cor: Cuba central-Cuba oriental

\begin{tabular}{lcccccc}
\hline Elevaciones & Indice (\%) & PC & Coc-Cc & CC & Cc-Cor & Total end./elev. \\
\hline Sierra del Chorrillo & 11.8 & 18 & 1 & 5 & 8 & 32 \\
Sierra de Najasa & 8.6 & 9 & 1 & 1 & 2 & 13 \\
Sierra de Guaicanámar & 2.1 & 3 & & & & 3 \\
Cerro Cachimbo (N) & 1.5 & 1 & & & & 1 \\
Cerro Cachimbo (S) & 3.7 & 2 & & & & 2 \\
\hline
\end{tabular}

Tabla 4 - Potencialidades económicas de la flora de las diferentes elevaciones. MD: medicinal, ML: melífera, MR: maderable, OA: otras aplicaciones.

\begin{tabular}{lcccccc}
\hline Elevaciones & Taxones & $\begin{array}{c}\text { \% representati-vidad } \\
\text { respecto a la flora del área }\end{array}$ & MD & ML & MR & OA \\
\hline Sierra del Chorrillo & 207 & 75.8 & 157 & 85 & 91 & 112 \\
Sierra de Najasa & 128 & 76.3 & 104 & 64 & 47 & 75 \\
Sierra Guaicanámar & 73 & 77.6 & 59 & 39 & 32 & 55 \\
Cerro Cachimbo (N) & 37 & 57.8 & 37 & 23 & 13 & 31 \\
Cerro Cachimbo (S) & 47 & 87.0 & 38 & 26 & 15 & 26 \\
\hline
\end{tabular}




\section{REFERÊNCIAS BIBLIOGRÁFICAS}

Arias, R. 1994. Arboles nativos de uso múltiple utilizados por pequeños productores de Guatemala. Revista Forestal Centroamericana 3(7): 10-15.

Avila, J.; Pérez, E.; Herrera, P.; Enríquez, N. \& Cárdenas, A. 1987. Estudio florístico y de vegetación de algunas elevaciones de la Sierra del Chorrillo, municipio Najasa, Camagüey. Instituto de Ecología y Sistemática, Filial Camagüey [inédito].

Barreto, A.; Herrera, P.; Enríquez, N. \& Espín, G. 1992. Lista florística de Monte Quemado, sureste de la Reserva Natural de la Sierra del Chorrillo, municipio Najasa, provincia de Camagüey, Cuba. Reporte de Investigación del Instituto de Ecología y Sistemática 9, 16p.

Barreto, A.; Enríquez, N.; Pérez, E.; Reyes, G.; Primelles, J. \& Sedeño, E. 2002. Aportes al conocimiento de la riqueza florística para la gestión ambiental de la Sierra de Najasa, Camagüey, Cuba. Rodriguesia 53(82): 135-149.

Borhidi, A. 1976. Fundamentos de Geobotánica en Cuba. Tesis para el grado a Doctor en Ciencias Biológicas, Budapest, 345p.

Borhidi, A. \& Muñiz, O. 1986. The phytogeographic survey of Cuba. II. Floristic relationships and phytogeographic subdivision. Acta Botanica Hungarica 32(1-4): 3-48.

Flores, J. C.; Martínez, C.; Olvera, M.; Galván, R. \& Chávez, C. 1988. Potencial de algunas leguminosas de la flora Yucatense como alimento humano y/o animal. Turrialba 38(2): 159-162.

Fors, A. J. 1957. Maderas cubanas. 4ta. ed. La Habana, 162p.

Fuentes, V. 1988. Las plantas medicinales de Cuba. Tesis para el grado a Doctor en Ciencias Biológicas, La Habana.

Havard-Duclos, B. 1969. Las plantas forrajeras tropicales. I. L., La Habana, 376p.

Hernández, J. \& M. E. López 1991. Lista preliminar de plantas tintóreas que crecen en Cuba. Revista Jardín Botánico Nacional 11(2): 133-144.

National Academy of Sciences. 1979. Tropical legumes: resources for the future. National Academy of Science, Washington DC., 100p.

Ordext, G. S. 1978. Flora apícola de la América tropical. Ed. Científico-Técnica, La Habana, 309p.

Pérez, E.; Enríquez, N. \& Oviedo, R. 1994. Características florísticas y fisionómicas de la vegetación boscosa de las sierras Najasa, Guaicanámar y Cerros Cachimbos, municipio Najasa, Camagüey, Cuba. Acta Botánica Cubana 95, 23p.

Primelles, J. \& colectivo de autores 1999. La aplicación de un SIG a la protección del bosque. CD Memorias Evento Trópico'99, Ciudad de La Habana, Cuba.

Reyes G. \& colectivo de autores 2002. CD Atlas multimedia de la flora medicinal potencial de la provincia de Camagüey. Centro de Investigaciones del Medio Ambiente, Camagüey, CITMA.

Roig, J. T. 1974. Plantas medicinales, aromáticas o venenosas de Cuba. Ed. Ciencia y Técnica, I. L., La Habana, 949p. 